\title{
Cold Particle Dark Matter
}

Kimmo Tuominen (D)

check for updates

Citation: Tuominen, K. Cold Particle Dark Matter. Symmetry 2021, 13, 1945 https://doi.org/10.3390/sym13101945

Academic Editors: Stefano Moretti and Rikard Enberg

Received: 6 September 2021

Accepted: 11 October 2021

Published: 15 October 202

Publisher's Note: MDPI stays neutral with regard to jurisdictional claims in published maps and institutional affiliations.

Copyright: (C) 2021 by the author. Licensee MDPI, Basel, Switzerland. This article is an open access article distributed under the terms and conditions of the Creative Commons Attribution (CC BY) license (https:// creativecommons.org/licenses/by/ $4.0 /)$.
Department of Physics, University of Helsinki, P.O. Box 64, FI-00014 Helsinki, Finland; kimmo.i.tuominen@helsinki.fi

Abstract: Possible dark matter candidates in particle physics span a mass range extending over fifty orders of magnitude. In this review, we consider the range of masses from a few keV to a few hundred $\mathrm{TeV}$, which is relevant for cold particle dark matter. We will consider models where dark matter arises as weakly coupled elementary fields and models where dark matter is a composite state bound by a new strong interaction. Different production mechanisms for dark matter in these models will be described. The landscape of direct and indirect searches for dark matter and some of the resulting constraints on models will be briefly discussed.

Keywords: dark matter; early universe; direct detection; indirect detection

\section{Introduction}

Cold Dark Matter (CDM) has emerged as an excellent parametrisation for $26 \%$ of the energy density of the universe in addition to the components parametrised as baryonic matter and dark energy [1]. In spite of these precision observations, the non-gravitational nature of dark matter (DM) remains enigmatic [2-4]. From cosmological observations concerning, on one hand, the light element abundance and, on the other hand, the cosmic microwave background (CMB) radiation spectrum, we can infer that the Standard Model (SM) degrees of freedom must have been in thermal equilibrium in the early universe [1,5-8]. Whether DM was ever part of the same heat bath is not known.

Theoretical possibilities for DM are manifold. This is concretely encapsulated by the fact that the masses of possible dark matter candidates extend from $10^{-20} \mathrm{eV}$ to the Planck mass $M_{\mathrm{Pl}}$ and beyond to the order of solar masses. This is illustrated schematically in Figure 1. In the case of ultralight dark matter [9] the dark matter arises in the form of collective field configurations with the de Broglie wave length of the typical galactic size. This form of dark matter may resolve problems in structure formation, but it is currently unclear if the problems are of physical origin or due to limitations in the current computational approaches to address dynamics of the formation of the large-scale structures in the universe. At the largest end of the mass spectrum, the possible candidates are dim objects composed of baryonic matter or microscopic black holes. The former are ruled out by microlensing observations while the latter are heavily constrained if they are required to constitute all of the observed dark matter abundance [10].

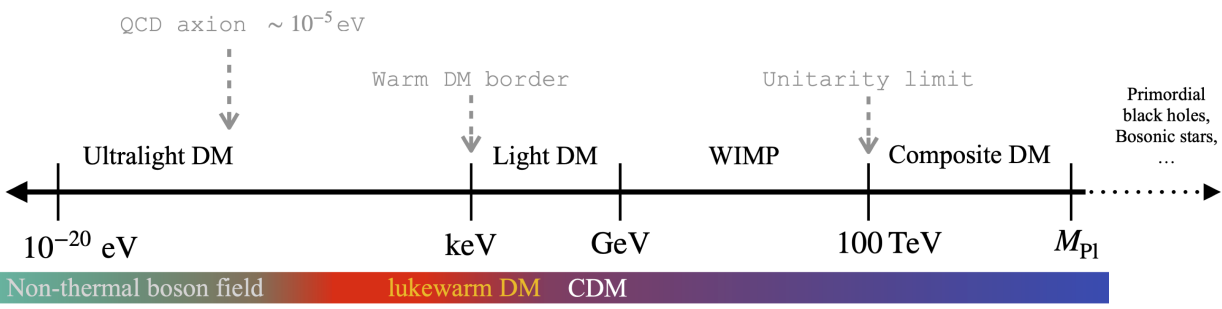

Figure 1. A schematic illustration of the range of possible masses of DM candidates. The color bar below the masses indicates the change from non-thermal to hot and warm and to cold DM in relation to the mass scale. 
In the mass range, starting at a few $\mathrm{keV}$, the dark matter is composed of non-relativistic, cold particles. On one hand, the existence of such CDM describes the observations at galactic scales, on the scale of galaxy clusters and on the scale of the entire observable universe. It should be noted, however, that ultimately the solution to the dark matter puzzle may lie within the theory of gravitation itself, as demonstrated by modified gravity models [11,12]. However, the relativistic generalization consistently describing the observations at different distance scales, extending from galactic distance scales to the entire universe, remains a challenge; see e.g., [13-15]. In this review, we will therefore not dwell on the topic of modified gravity further. On the other hand, the CDM paradigm is appealing in light of our present understanding of ordinary matter, as described by the Standard Model (SM) of elementary particle interactions. The CDM paradigm puts dark matter within the framework of beyond-SM models and opens up prospects for experimental searches for DM in dedicated direct search experiments, collider experiments and in the analysis of various astrophysical probes.

The modeling of particle DM depends on the assumed mechanisms of its production in the early universe. If we assume that the dark matter abundance is borne out as a thermal relic, this leads to two general mechanisms [16,17]. Namely, if the DM was in thermal equilibrium with the SM fields, the freeze-out mechanism may explain its present abundance. This occurs via thermal decoupling when the DM annihilation rate falls below the expansion rate of the early universe. The second main possibility is that of freeze-in. In this case, the DM was never in thermal equilibrium but its phase space density is populated from the thermal SM bath due to very weak but nonzero couplings between SM fields and DM. There are various mechanisms refining these two categories or combining their essential ideas, which we will discuss in more detail in later sections of this review.

However, thermal origin may not be a justified assumption. After all, the stable particle of the SM and constituting the visible matter, i.e., the proton, did not obtain its abundance in this way. It is possible to obtain the DM abundance via 'darkeongenesis' similar to the ordinary baryons [18]. It is a very appealing scenario in which the abundances of light and dark matter would be intrinsically interconnected by the symmetries of the relevant extension of the SM.

The different production mechanisms of dark matter are also connected with the precise nature of the DM candidate. In models with weakly coupled elementary particles for which perturbation theory provides an adequate tool to carry out theory and phenomenology analyses, the thermal relic paradigm provides a framework in which controlled computations can be carried out and used for phenomenology studies. On the other hand, asymmetric dark matter arises more naturally in the framework in which DM arises as composite states of strongly coupled elementary constituents [19]. Here, the analyses must be carried out using effective theories and, if feasible, first principle lattice computations. These features are also interconnected with the question of the self interactions in DM, and we will separately discuss the cases of weakly coupled thermal relics and strongly coupled composite dark matter. Of course, the thermal origin of DM abundance can be attributed to composite DM as well [20] and, similarly, fundamental fields can constitute asymmetric dark matter [21].

The production mechanisms of DM are also connected with other particle physics processes. For example, particle annihilation also implies particle production, which may be relevant for current and future collider experiments and provide important constraints for models. Furthermore, the particle interactions relevant for production of DM also imply DM scattering cross sections with ordinary matter, which can be probed in dedicated experiments. Finally, the possibly nonzero cross sections of dark matter scattering on itself are also important for understanding the formation and evolution of galactic halos and the cosmic collisions of galaxies and galaxy clusters.

Before the discovery of the Higgs, much of the theoretical work in beyond SM phenomenology was focusing on the need to explain the origin of the electroweak scale. In this type of model, the dark matter arises within the extended particle content and is 
naturally connected with the electroweak sector as, for example, the neutralino in the supersymmetric extension of the minimal supersymmetric SM or a bound state of new strongly interacting degrees of freedom underlying the composite Higgs sector.

However, with the discovery of the SM-like Higgs boson at LHC [22,23] and no extended spectrum of excitations, the dark matter problem was placed into focus as the motivation to extend the SM. This paradigm shift has resulted in active research on the extensions where the isolated SM is connected to an isolated dark sector via some restricted set of messenger particles. For DM phenomenology, such a paradigm change was very welcome: first, it allows for a generalization of DM models beyond simple scenarios of weakly interacting massive particles (WIMPs) such as supersymmetric DM. Second, it leads to well-defined benchmarks for experimental testing. Third, it allows for a wider exploration of DM phenomenology connected more with the experimental searches and data rather than purely theoretical premises such as naturality or the strong CP problem.

These facts are manifested by the recent developments in the field of observations relating to DM. There are many astrophysical and cosmological observations as well as an active experimental program of direct detection experiments contributing to constraining various hidden sector models. As a motivation for isolated dark matter, there is one observation which stands out; the observations on colliding galaxy clusters [24], although there are also caveats [25]. Nevertheless, dark matter decoupled from or interacting only very weakly with the SM fields implies the observed behavior. Moreover, the observation of the separation of the dominant sources of gravitational potential and radiating matter can be used to set bounds on the self-interactions of the dark matter degrees of freedom [26]. Such self-interactions affect the internal dynamics of dark sectors and have observable consequences [27].

In this review, we will follow, in more detail, the story outlined above, focusing on the mass range relevant for cold particle dark matter. We will first, in Section 2, consider the dark matter genesis in the case of weakly coupled thermal relic dark matter. The main mechanisms to be discussed here are freeze-out and freeze-in mechanisms. Then, in Section 3, we will consider the general landscape of dark sector models in some more detail emphasizing the possibilities of how the dark sector couples with the SM. In Section 4, we will consider strongly coupled composite dark matter, review its main motivations and discuss main results. In Section 5, we will briefly consider the observational and experimental landscape and the present constraints as well as some future developments. We will conclude and summarize in Section 6.

\section{Weakly Coupled Thermal Relics}

The CMB observations by the Planck satellite [1] show that the relic abundance of $\mathrm{CDM}$ is

$$
\Omega_{\mathrm{DM}} h^{2}=0.120 \pm 0.001,
$$

where $h$ is the present rate of expansion of the universe in units of $100 \mathrm{~km} / \mathrm{s} / \mathrm{Mpc}$ and $\Omega_{\mathrm{DM}}$ is the present DM energy density. The main question for DM modeling is to explain how does this relic abundance arises during the course of the evolution of the universe.

One major paradigm of particle DM is that its abundance is set by thermal equilibration and decoupling in the early universe. In order not to generate too large abundance, the relic must be cold, i.e., heavy with respect to the temperature where its decoupling occurs. Then its number density is suppressed, $n \sim(m T)^{3 / 2} \exp (-m / T)$. The DM freezes out as the interaction rate and the expansion rate of the universe become comparable, $n \sigma \sim H$. As is well known, the right magnitude of the DM abundance can be produced by $m \sim 10^{2} \mathrm{GeV}$ and $\sigma \sim G_{F}^{2} m^{2} \sim 10^{-8}$, where $G_{F}$ is the Fermi coupling. The coincidence with the typical electroweak cross section and mass around the electroweak scale became known as the 'WIMP miracle'. It should be noted that this needs to have nothing to do with the weak interaction, but given a cross section $\sigma \sim g^{4} / m^{2}$ with some coupling $g$ merely suggests a relation

$$
g^{2} \sim \frac{m}{10 \mathrm{TeV}^{\prime}}
$$


and the mass scale $m$ can be whatever. This type of general freezeout scenarios of WIMPs have received a lot of attention both by the theory and experimental communities.

Cosmic abundance can also be explained by populating the phase space of the DM candidate by decays and scatterings of SM particles with only tiny interaction with the DM degrees of freedom. This feebly interacting massive particle (FIMP) $[28,29]$ scenario is another alternative, where the DM particle needs not thermally equilibrate with the SM heat bath. In comparison to WIMPs, which require a sufficiently large interaction strength, making them discoverable in direct searches, the feeble interactions of FIMPs effectively hide them in direct searches and other detection methods must be applied.

\subsection{Computing the Relic Density}

The computation of the DM abundance in these scenarios is based on following the evolution of the particle phase space distribution functions. To illustrate, consider a single DM particle denoted by $\chi$ and its phase space distribution function $f_{\chi}(p, t)$. The time evolution in the expanding universe is given by the Boltzmann equation [30]

$$
\left(\partial_{t}-H \mathbf{p}_{\chi} \cdot \nabla_{\mathbf{p}}\right) f_{\chi}(p, t)=\sum_{j} \frac{1}{E_{\chi}} \int d C_{\chi, j}
$$

where $t$ is the time, $H$ is the Hubble parameter, $\mathbf{p}_{\chi}$ is the physical momentum and $E_{\chi}$ is the energy of $\chi$ particles. The sum on the right hand side is over all processes involved. The collision term $d C_{\chi}$ for the process $\chi+a_{1}+a_{2}+\cdots+a_{n} \rightarrow b_{1}+b_{2}+\cdots+b_{n}$ is

$$
\begin{aligned}
d C_{\chi}= & -\prod_{i} d \Pi_{a_{i}} \prod_{j} d \Pi_{b_{j}}(2 \pi)^{4} \delta^{(4)}\left(p_{\chi}+\sum_{k} p_{a_{k}}-\sum_{\ell} p_{b_{\ell}}\right) \times \\
& \left(|M|_{F}^{2} f_{\chi} f_{a_{1}} \cdots\left(1 \pm f_{b_{1}}\right)\left(1 \pm f_{b_{2}}\right) \cdots-|M|_{B}^{2} f_{b_{1}} f_{b_{2}} \cdots\left(1 \pm f_{\chi}\right)\left(1 \pm f_{a_{1}}\right) \cdots\right)
\end{aligned}
$$

where the subscript $F(B)$ is a shorthand for the forward process $\chi+a_{1}+a_{2}+\cdots+a_{n} \rightarrow$ $b_{1}+b_{2}+\cdots+b_{n}$ (and its inverse). The averaging in the squared transition amplitudes is taken over both the initial and final states. The usual phase space measure is

$$
d \Pi_{j}=\frac{g_{j}}{(2 \pi)^{3}} \frac{d^{3} p_{j}}{2 E_{j}}
$$

where $g_{j}$ is the number of intrinsic degrees of freedom of particle $j$ and $p_{j}$ denotes the momentum of the particle and $E_{j}$ its energy. Finally, the phase space distribution of each particle involved is denoted by $f_{j}$ and the \pm signs correspond to bosons and fermions.

Generally the above coupled equations determine the distribution functions of all particles involved. However, if all other particle species than $\chi$ are assumed to be in thermal equilibrium, the equations collapse to a single equation determining the distribution $f_{\chi}$. With given initial conditions and known particle interactions the solution of the Boltzmann equation can be integrated to determine the DM relic abundance.

Based on the above discussion we can now list different mechanisms for producing the observed DM abundance in this context. First, there is the freeze-out mechanism, where DM decouples from the visible sector heat bath as the expansion rate of the universe wins over the interaction rate of DM and heat bath particles. Consequently, the comoving number density of DM particles freezes to a constant value. As already mentioned, for a weak scale mass DM particle this requires a coupling of the order of the weak interaction, $g \sim \mathcal{O}(0.1)$, but other values are possible depending on the mass $m_{\chi}$.

Second, there is the freeze-in mechanism which is based on the assumption that the DM phase space is initially unpopulated, but subsequently produced from the visible sector by decays or annihilations. The DM abundance freezes to a constant value as the expansion rate of the universe wins over the DM production rate and DM does not need to enter thermal equilibrium at any stage. This requires a very small coupling, $g \sim \mathcal{O}\left(10^{-10}\right)$ 
between the visible sector and DM in order for the DM to remain isolated from the SM thermal bath and produce the correct relic abundance.

Third, the dark sector can have rich dynamics as combinations of the previous two mechanisms. For example if dark matter has self interactions, it can, after initially populated by freeze-in from the visible sector, constitute a heat bath within itself but decoupled from the visible sector. The temperatures of the two can be different and DM undergoes a dark freeze-out involving only the dark sector fields. Yet another scenario where the dark sector thermalizes on its own, but the dark freeze-out occurs while the yield from the visible sector is still ongoing. Then due to the sustained particle production, the DM annihilations within the dark sector resume and the final relic abundance is determined only after the yield has finally ended. This scenario has been dubbed as the reannihilation mechanism.

\subsection{Freeze-Out}

We will now illustrate some of the mechanisms enlisted in the previous subsection. Consider an extension of the SM by a stable particle $\chi$. A concrete and much studied example would be to take $\chi$ to be a real singlet scalar with a $Z_{2}$-symmetry coupled with the SM via interaction $\chi^{2}|H|^{2}$ [31-33].

To discuss the usual freeze-out mechanism, we assume no initial asymmetry between particles and anti-particles and we neglect any large self-interactions. Then, the abundance of DM is set by considering simply annihilation of $\chi$ into SM particles, $\chi \chi \rightarrow \mathrm{SM}$, and its inverse. Integrating the Boltzmann equation over momentum, gives the Zeldovic-OkunPikelner-Lee-Weinberg equation $[34,35]$ for the DM number density $n_{\chi}$,

$$
\frac{d n_{\chi}}{d t}+3 H n_{\chi}=-\left\langle v \sigma_{\chi}\right\rangle\left[n_{\chi}^{2}-\left(n_{\chi}^{\mathrm{eq}}\right)^{2}\right],
$$

where $\left\langle v \sigma_{\chi}\right\rangle$ is the thermal average of the total DM annihilation cross section times the velocity [36], $n_{\chi}$ is the DM number density we want to determine and $n_{\chi}^{\mathrm{eq}}$ is the number density at equilibrium.

It is common to switch to dimensionless variables, $Y=n_{\chi} / s$ and $x=m_{\chi} / T$, defined in terms of the mass of the DM particle, $m_{\chi}$, entropy density $s$ and temperature $T$ of the visible sector. Then the above equation becomes

$$
\frac{x}{Y_{\mathrm{eq}}} \frac{d Y}{d x}=-\frac{\Gamma}{H}\left[\left(\frac{Y}{Y_{\mathrm{eq}}}\right)^{2}-1\right],
$$

where we have assumed that the number of relativistic degrees of freedom remains constant and defined $\Gamma=n_{\chi}^{\mathrm{eq}}\left\langle v \sigma_{\chi}\right\rangle$.

This equation operates as follows: as long as the expansion rate $H$ remains smaller than the interaction rate $\Gamma$, the DM particles remain in equilibrium with the SM particles and the particle yield $Y$ traces the equilibrium yield, $Y \simeq Y_{\mathrm{eq}}$. Then, as the expansion rate becomes comparable to the interaction rate, the annihilations become ineffective and the DM abundance freezes out. Assuming that the DM is nonrelativistic at the freeze-out, the present-day DM abundance is approximated as [30]

$$
\Omega_{\chi} h^{2} \simeq 5.36 \times 10^{9}(n+1) \frac{\sqrt{g_{*}}}{g_{* s}} \frac{m_{\chi}}{T_{f}} \frac{\mathrm{GeV}^{-1}}{M_{\mathrm{Pl}}\left\langle v \sigma_{\chi}\right\rangle},
$$

where $g_{*}$ and $g_{* s}$ denote, respectively, the effective numbers of relativistic degrees of freedom in energy and entropy densities and $T_{f}$ is the freeze-out temperature. The parameter $n=0$ for $s$-wave annihilation, and $n=1$ for $p$ wave annihilation. This simple DM freeze-out scenario has notable refinements, such as the coannihilation, the annihilation into forbidden channels and the annihilation near poles [37], yet further scenarios arise in the presence of semi-annihilations [38] and the co-scatterings [39]. 
The important feature in the above equation is that the present abundance is inversely proportional to the DM annihilation cross section. This is intuitive, since the stronger the interactions, the longer the DM will remain in equilibrium with the SM and, consequently, more diluted its abundance will become before finally freezing out.

\subsection{Freeze-In}

The above analysis was based on the starting point that the DM initially is in equilibrium with the SM. To relax this assumption, one takes the DM to interact with the SM so weakly that it cannot come into equilibrium. The couplings required are of the magnitude $\mathcal{O}\left(10^{-7}\right)$ or smaller [40] depending on the explicit model. As a simple example, consider the case where the abundance of the DM particle $\chi$ is initially zero, but can be produced by the decays of the heat bath particles $[28,29,41]$, for example $h \rightarrow \chi \chi$ where $h$ is an SM particle coupled with the DM, for example, the Higgs. The DM abundance freezes in as the number density of $h$ becomes Boltzmann suppressed.

In such a case, assuming that $h$ obeys Maxwell-Boltzmann statistics, the Boltzmann equation for the DM number density is approximated as

$$
\frac{d n_{\chi}}{d t}+3 H n_{\chi}=2 \Gamma_{h \rightarrow \chi \chi} \frac{K_{1}(x)}{K_{2}(x)} n_{h}^{\mathrm{eq}},
$$

where $\Gamma_{h \rightarrow \chi \chi}$ is the decay width and $n_{h}^{\mathrm{eq}}$ is the equilibrium number density of $h$. We also denoted $x=m_{h} / T$ and the functions $K_{j}(x)$ are the modified Bessel functions of the second kind.

Again, defining $Y=n_{\chi} / s$ and assuming a constant number of relativistic degrees of freedom, we can rewrite the above equation as

$$
\frac{x}{Y_{h}^{\mathrm{eq}}} \frac{d Y}{d x}=2 \frac{\Gamma_{h \rightarrow \chi \chi}}{H} \frac{K_{1}(x)}{K_{2}(x)} .
$$

The approximate solution is [29]

$$
\Omega_{\chi} h^{2} \simeq 4.48 \times 10^{8} \frac{g_{h}}{g_{* s} \sqrt{g_{*}}} \frac{m_{\chi}}{\mathrm{GeV}} \frac{M_{\mathrm{Pl}} \Gamma_{h \rightarrow \chi \chi}}{m_{h}^{2}},
$$

where $g_{h}$ is the number of intrinsic degrees of freedom of the $h$ field and we assumed $T \simeq m_{h}$. Let us denote the coupling between $h$ and $\chi$ by $y$ so that $\Gamma_{h \rightarrow \chi \chi} \simeq y^{2} m_{h} /(8 \pi)$. Then, assuming that $g_{* s} \simeq g_{*}$, we obtain and estimate for the magnitude of the coupling $y$ needed to produce the required DM abundance,

$$
y \simeq 10^{-12}\left(\frac{\Omega_{\chi} h^{2}}{0.12}\right)^{1 / 2}\left(\frac{g_{*}}{100}\right)^{3 / 4}\left(\frac{m_{h}}{m_{\chi}}\right)^{1 / 2} .
$$

This is consistent with the fact that we assumed a very weak coupling in the beginning of the analysis. We also see that the parametric behavior with respect to the increased coupling is opposite to that of freeze-out: here, increased interaction rate implies increased abundance.

If the freeze-in abundance is produced by $2 \rightarrow 2$ scatterings, say from operator $y \chi h_{1} h_{2} h_{3}$ rather than decays, the final abundance becomes [29]

$$
\Omega_{\chi} h^{2} \simeq 1.01 \times 10^{24} \frac{y^{2}}{g_{* s} \sqrt{g_{*}}}
$$

again, under the assumption that $T \simeq m_{\chi}$ and that the masses of the $h_{i}$ particles can be neglected over the mass of $\chi$. Also from this we find that, to generate a large enough DM, abundance requires 


$$
y \simeq 10^{-11}\left(\frac{\Omega_{\chi} h^{2}}{0.12}\right)^{1 / 2}\left(\frac{g_{*}}{100}\right)^{3 / 4} .
$$

In addition to the differences between the required interaction strength and initial abundances between the freeze-out and freeze-in, the relation between the temperature and the relevant energy scale $m$ is different. Typically the frozen-out relic abundance is produced at $m_{\chi} / T \simeq 10 \ldots 30$, while this ratio is $m_{\chi} / T \simeq 2 \ldots 5$ in the case of freeze-in.

Here we have assumed that the initial number density of DM particles is negligible. This can be affected by the initial conditions set by cosmic inflation [40,42-44]. Such sensitivity does not arise in the freeze-out scenario, as thermal equilibrium destroys all dependence on initial conditions. In freeze-in, sensitivity to effects of non-renormalizable operators may also arise [29,45-47].

\subsection{Dark Freeze-Out and Reannihilation}

Let us then consider another of the initial assumptions; namely, that the interactions in the dark sector can be neglected. If the interactions in the dark sector are sufficiently large, then after being produced by freezing-in from the visible sector, the DM particles may thermalize within the dark sector, independent of the visible sector. If this happens, then the final abundance is not set by the initial freeze-in but by the subsequent dark freeze-out occurring solely within the dark sector [27,48-51].

Chemical equilibration in the dark sector can be achieved by sufficiently fast number changing processes, which reduce the average momentum of DM particles and simultaneously increase their number density. For example, consider self-interactions $\lambda \chi^{4}$ for a single species of dark sector particles $[27,49,50]$. Then the $2 \rightarrow 4$ scatterings lead to thermalization if the self-coupling exceeds a critical value $\lambda_{c}$; for values below this, the usual freeze-in framework is sufficient. If the couplings are large enough, the dark sector enters thermal equilibrium with temperature $T_{\mathrm{D}}$, which is different from the visible sector temperature. At this stage, the $2 \rightarrow 4$ processes and their inverses occur at the same rate. As the DM cools, the $2 \rightarrow 4$ scatterings shut off but the equlibrium number density is maintained by the inverse annihilations. During this cannibalization era, the average momentum of the DM particles increases while their number density decreases. Finally, the annihilation rate becomes small enough and the number density freezes out. For further details on these dynamics, see, e.g., [27,50-54].

As another refinement of these thermal dynamics, we mention reannihilation $[50,53,55,56]$. This scenario is akin to the dark freezeout, but the yield from the SM has not shut off before the dark sector reaches thermal equilibrium. The dark freezeout, which otherwise would occur, is prevented by the increase in the particle yield from the SM freeze-in processes. As a function of time, the DM number density first traces the equlibrium number density, then the so-called quasi-static equilibrium density and at the intercept of a critical value $Y_{\mathrm{c}} \simeq\langle v \sigma\rangle s Y_{\text {eq }}^{2} / H$ the number density finally freezes out; see [50].

As these examples show, the interactions within the dark sector can lead to interesting new mechanisms for particle production beyond the simplest freeze-out and freeze-in scenarios. The key role is played by sufficiently strong self-interactions. Since these occur naturally within the scope of strongly interacting composite dark matter, we will return to these considerations in Section 4. In the next section, we will discuss in more detail the models for generic dark sectors and their coupling with the SM.

\section{Dark Sectors and Portals}

As illustrated by the WIMP miracle, and more generally by the solutions of the Boltzmann equation for the DM relic abundance in the early universe, to set the correct abundance via annihilations or particle decays in the early universe requires the existence of some mediator particle coupling DM with the SM fields. To dial for the correct relic density and to suppress the scatterings of DM on ordinary matter so that DM has escaped 
numerous laboratory experiments carried out so far, the mass of the mediator and its couplings need be tuned appropriately.

This setting motivates the framework of dark sectors, where the DM and possibly other degrees of freedom reside, and which are separated from the SM and interact with ordinary matter only via specific portals. There are numerous possibilities how this setting is realized. In the simplest case the DM is just a real singlet scalar coupled with SM via the Higgs, and, in this case, the DM and its interactions are mediated by the same particle. Another minimal setting would be a dark gauge field, possibly with a fermion charged under this new interaction. Examples of more complex realization would be dark sectors with multitude of states akin to the particle content of the SM itself.

\subsection{Cosmology of Hidden Sectors}

The essential model building feature is the linking of the dark sector with the SM. Then, a natural question to ask is how well-motivated such a link is or whether it is really needed at all. To address this possibility, let us consider a dark sector consisting of a fermion $\chi$ singlet under all SM interactions, but charged under a new interaction mediated by a vector field $V$. Let us assume that $m_{\chi}>m_{V}$ and consider annihilation $\chi \chi \rightarrow V V$. Then, the thermal freeze-out can progress in the hidden sector without the interactions with the SM needed to arise at all.

To investigate this scenario further, let us assume that the dark sector was initially populated during the reheating phase of the early universe, leading to a thermal plasma of dark sector states not interacting with the SM and having a temperature $T_{\mathrm{D}}$ of its own. The difference in the SM and hidden sector temperatures is parameterized as

$$
\xi \equiv \frac{T_{\mathrm{D}}}{T}
$$

which is a function depending on time. The relic abundance arises via the freeze-out scenario where, in the simplest case, the thermal population of the vector field $V$ with $m_{V} \ll m_{\chi}$ plays the role of the relativistic thermal bath at temperature $T_{\mathrm{D}}$.

However, the entropy and energy density initially stored in the dark sector must go somewhere. For a stable $V$, the number density of its relic density scales as $\xi^{3} T^{3}$. For and order one value of $\xi$, it turns out that in order not to generate overabundance of CDM or a sizable quantity of hot DM, $V$ must behave as an additional component of radiation in the early universe which can be constrained by observations [57,58].

The effects of such new radiation component is usually represented in terms of the effective number of relativistic degrees of freedom, $N_{\text {eff }}$. To see how this is constrained, consider the total energy density of radiation

$$
\rho_{\mathrm{T}}=\frac{2 \pi^{2}}{30} T^{4}+\frac{7 N_{\mathrm{eff}, \mathrm{SM}}}{4} \frac{\pi^{2}}{30} T_{v}^{4}+\rho_{\mathrm{D}}
$$

where we included the photons and neutrinos of the SM (as the first and second terms, respectively) and the contribution from the dark sector $\left(\rho_{\mathrm{D}}\right)$. The dark sector energy density is

$$
\rho_{\mathrm{D}}=g_{\mathrm{D}} \frac{\pi^{2}}{30} \xi^{4} T^{4}
$$

where $g_{\mathrm{D}}$ counts the degrees of freedom in the dark sector at temperature $T_{\mathrm{D}}$. In SM, the value of $N_{\text {eff,SM }}$ is $3.046[29,59,60]$, and assuming that the neutrino temperature is as in the $\mathrm{SM}$, we can represent the total energy density as

$$
\rho_{\mathrm{T}}=\rho_{\gamma}+\left(N_{\mathrm{eff}, \mathrm{SM}}+\Delta N_{\mathrm{eff}}\right) \frac{7}{4} \frac{\pi^{2}}{30} T_{v}^{4} .
$$

Here, we denoted the component of the photons by $\rho_{\gamma}$ and defined $\Delta N_{\text {eff }}$ as the deviation in $N_{\text {eff }}$ from the SM value. Hence, we obtain the constraint 


$$
\Delta N_{\mathrm{eff}}=\frac{4}{7} g_{\mathrm{D}} \xi^{4} \frac{T^{4}}{T_{v}^{4}}
$$

This is a time-dependent quantity, and the constraints from the big bang nucleosynthesis and $\mathrm{CMB}$ are from specific epochs: the former concerns the era $T \sim 10 \mathrm{keV}-1 \mathrm{MeV}$ while the latter the era $T<\mathrm{eV}$. The results from [1,61] are that $N_{\text {eff,BBN }}=2.89 \pm 0.28$ and $N_{\text {eff,CMB }}=2.99 \pm 0.17$. It should be noted that assumptions about, e.g., neutrino masses or DM self-interactions, can significantly affect the error bars quoted above.

In any case, the above consideration, leading to the constraint $\Delta N_{\text {eff }} \sim 0.5$ shows that in principle decoupled dark sectors can be constrained through gravitational effects alone. This argument is expected to become more refined as the sensitivity of observations is increased. For the $\Delta N_{\text {eff }}$ constraints in various models, see e.g., [62-64].

Another probe into the dynamics of dark sectors decoupled from the visible one are provided by possible dark sector self-interactions as they affect the clustering of DM. Sufficiently strong self-scattering of DM can lead to DM thermalization in galactic halos and reduction in the central densities within the cores of galaxies. The motivation for DM self-interactions arises from the so called 'cusp vs core' problem [65-70], i.e., observation of shallowness of the central profiles of dwarf galaxies in comparison to the simulations, and the missing satellites problem, i.e., observation of far too few satellites of the Milky Way, and the related 'too-big-to-fail' problem [71,72]. It should be noted that improvements in the simulations, in particular more proper inclusion of baryonic physics [73-78], may explain the dwarf halo profiles and more satellites are being discovered. Nevertheless, the DM self interactions remain as an important generic property of DM, deserving further study.

The importance of DM self interactions is characterized by the transfer cross section,

$$
\sigma_{\mathrm{T}}=\int d \Omega \frac{d \sigma}{d \Omega}(1-\cos \theta)
$$

over the DM mass and its typical value

$$
\frac{\sigma_{\mathrm{T}}}{m_{\chi}} \sim 1 \frac{\mathrm{cm}^{2}}{g}=2 \cdot 10^{-24} \mathrm{~cm}^{2}\left(\frac{\mathrm{GeV}}{m_{\chi}}\right) .
$$

For DM density, $\rho_{\mathrm{D}} \simeq \mathrm{GeV} / \mathrm{cm}^{3}$, and velocity $v \sim 10^{-3}$, the scattering rate is

$$
\Gamma \simeq \frac{\rho_{\mathrm{D}}}{m_{\chi}} \sigma_{\mathrm{T}} v \simeq 10^{-9} \frac{1}{\text { year }} .
$$

The dynamical time scale for a typical galaxy (such as the Milky Way) and its DM halo is about $10^{8}-10^{9}$ years, so the above value of the transfer cross section is about what is needed to affect the galactic dynamics. Of course, this is just an order of magnitude and significantly higher interaction rates can arise, e.g., in the inner regions of the galactic halo.

Since no significant offset in the mass distribution of DM in colliding galaxy clusters was observed, this translates into an upper bound for DM self-interactions. For example, the Bullet Cluster constrains such aself-interacting cross section by $\sigma / m<1.25 \mathrm{~cm}^{2} / \mathrm{g}$ at $68 \%$ CL $[24,26,79]$. Observations of several cluster collisions provide the constraint $\sigma / m<$ $0.47 \mathrm{~cm}^{2} / \mathrm{g}$ at $95 \%$ CL [80]. This bound is dependent on the model assumptions [81-84] and relaxing these would allow for a less-stringent bound.

On the other hand, in the galaxy cluster, Abell 3827, an offset between luminous matter and the DM halo has been observed and if interpreted as an effect due to DM selfinteractions, this implies a non-vanishing $\sigma / m \sim 10^{-4} \mathrm{~cm}^{2} / \mathrm{g}$ [85]. The analysis in [86,87] leads to values $\sigma / m \sim 1.5 \mathrm{~cm}^{2} / \mathrm{g}$.

To see how the bound on the DM self-interactions constrains models, let us return to the model we considered earlier and assume an ultralight mediator $m_{V} \ll \mathrm{eV}$ and 
$m_{\chi} \gtrsim \mathrm{keV}$. Then, in particular $m_{V} \ll m_{\chi} v$, and the transfer cross section is enhanced in forward scattering and is given by

$$
\sigma_{\mathrm{T}} \simeq \frac{8 \pi \alpha_{\chi}^{2}}{m_{\chi}^{2} v^{4}} \ln \frac{m_{\chi}^{2} v^{2}}{m_{V}^{2}}
$$

with the bound $\sigma_{\mathrm{T}}<1 \mathrm{~cm}^{2} / \mathrm{g}$, this implies that

$$
\alpha_{\chi} \lesssim 10^{-10}\left(\frac{v}{10^{-3}}\right)^{2}\left(\frac{m_{\chi}}{\mathrm{MeV}}\right)^{3 / 2}\left(\frac{10}{\ln \left(m_{\chi}^{2} v^{2} / m_{V}^{2}\right)}\right)^{1 / 2},
$$

On the other hand, in this model, the relic density is determined by the annihilation process $\chi \chi \rightarrow V V$ whose cross section is

$$
\langle\sigma v\rangle \simeq \frac{\pi \alpha_{\chi}^{2}}{m_{\chi}^{2}}
$$

To obtain the correct relic density requires $\langle\sigma v\rangle \sim 5 \cdot 10^{-9} / \mathrm{GeV}^{2}$. For example, with $m_{\chi} \gtrsim \mathrm{keV}$, this implies that $\alpha_{\chi} \gtrsim 4 \cdot 10^{-11}$ and we see that the sufficiently strongly interacting light dark matter excludes thermal freeze-out.

There are various ways to bypass the limitations arising in the simplest model frameworks. For example, one can introduce additional states into the hidden sector to facilitate thermal freeze-out without introducing the ultralight limit for the mediator $V$. If $m_{V} \gg m_{\chi} v$, then the self interaction cross section is $\sigma_{\mathrm{T}} \simeq \pi \alpha_{\chi}^{2} m_{\chi}^{2} / m_{V}^{4}$. The resulting constraints do not exclude the possibility of thermal freeze-out.

It should also be noted that the above simple discussion takes into account the cross sections in the limit of the Born approximation. Generally, there are resonant enhancement effects requiring a solution of the general quantum mechanical scattering problem. For further details, see $[88,89]$. As another remark, DM scattering with sufficiently strong DM self-interactions and dark radiation can lead to dark acoustic oscillations; see, e.g., [90,91].

In this section, we have made the assumption that the dark sector has no coupling with the visible one, since we wanted to illustrate that even in such case there are strong observational constraints. However, in many dark sector models, this is not the case.

\subsection{Directly Coupled Dark Sectors}

To illustrate the opposite situation to the one considered in the previous subsection, let us now assume that DM and SM degrees of freedom are in thermal equilibrium. The DM annihilations into SM particles redistribute the energy density initially deposited in the dark sector into the SM thermal bath. If DM is sufficiently heavy, the $N_{\text {eff }}$ bounds from nucleosynthesis and $\mathrm{CMB}$ discussed in the previous subsection are alleviated.

However, if the mass scale of $\mathrm{DM}$ is close to the $\mathrm{MeV}$ scale so that the thermal freezeout happens near the time of the nucleosynthesis there will be observational consequences. For example, if DM is in equilibrium with the electron positron plasma after neutrinos have decoupled then electron-positron annihilation will contribute to heating of DM and consequently temporarily reduces the energy density stored in photons. This, in turn, increases the baryon-to-photon ratio, and this increase allows for deuterium to convert into helium efficiently. Observations lead to bound $m_{\chi} \gtrsim 1-10 \mathrm{MeV}[92,93]$.

Additionally, the CMB can be affected by DM annihilations. After freezeout, the DM annihilation in principle continues through the epoch of recombination. Even if the rate is negligible in relation to the changes in the relic density, the $\mathrm{CMB}$ is sensitive to the resulting energy injection. The main effect is an increase in residual ionized hydrogen. The energy injection is described in terms of the efficiency $f(z)$ characterizing the efficiency of energy deposition at redshift $z$ [94], and in the recent Planck results [1], the bounds are quoted in terms of an effective efficiency $f_{\text {eff }}$, defined as the leading coefficient in a particular 
functional basis suited to systematically expand the effect on the CMB . For the s-wave annihilation, the result from [1] is given in terms of an effective parameter

$$
p_{\mathrm{ann}} \equiv f_{\mathrm{eff}} \frac{\langle\sigma v\rangle}{m_{\chi}} \lesssim \frac{3 \cdot 10^{-26} \mathrm{~cm}^{3} / \mathrm{s}}{100 \mathrm{GeV}} .
$$

If compared with the thermal relic cross section for DM annihilation, for example, for annihilation into SM fermions, this excludes masses below $\mathcal{O}(10 \mathrm{GeV})$.

The direct coupling between DM and the SM fields can also be probed using various astrophysical observations. A notable signal is the Galactic center gamma ray excess [95-101]. While the signal could have its origin in astrophysical processes-see, e.g., [102-107]-DM annihilation is a viable possibility—see, e.g., [108,109]—but it is in tension with recent observations [110].

In addition to gamma rays, other particle fluxes have also been measured. The ratio of antiproton and proton fluxes measured by AMS-02 experiment, exhibits an excess over the expected background [111]. This can be interpreted as a signal of DM annihilations [112-119]. In some of these analyses, DM annihilations can explain excess both in the AMS antiproton data and in the galactic center gamma rays-see e.g., [120]. It should be noted that, within systematic errors, secondary astrophysical production alone can account for the data [121]. As a final example, an excess in high-energy cosmic positrons has been observed by PAMELA [122] and subsequently by AMS-02 [123]. It should be kept in mind that plausible explanations may arise from standard astrophysical objects [124,125]. Explanations by DM annihilations have also been pursued [126,127]), although these are at odds with the constraint from CMB [1].

The results discussed above are summarized in Figure 2 from [1] where solid straight lines show the $\mathrm{CMB}$ constraints on different annihilation channels and different regions show the AMS proton excess, galactic center gamma ray excess, as indicated. Additionally, the AMS/PAMELA positron fraction and electron and positron fluxes from Fermi/H.E.S.S. observations are shown as indicated by the figure. For further detail, we refer to the original reference [1].

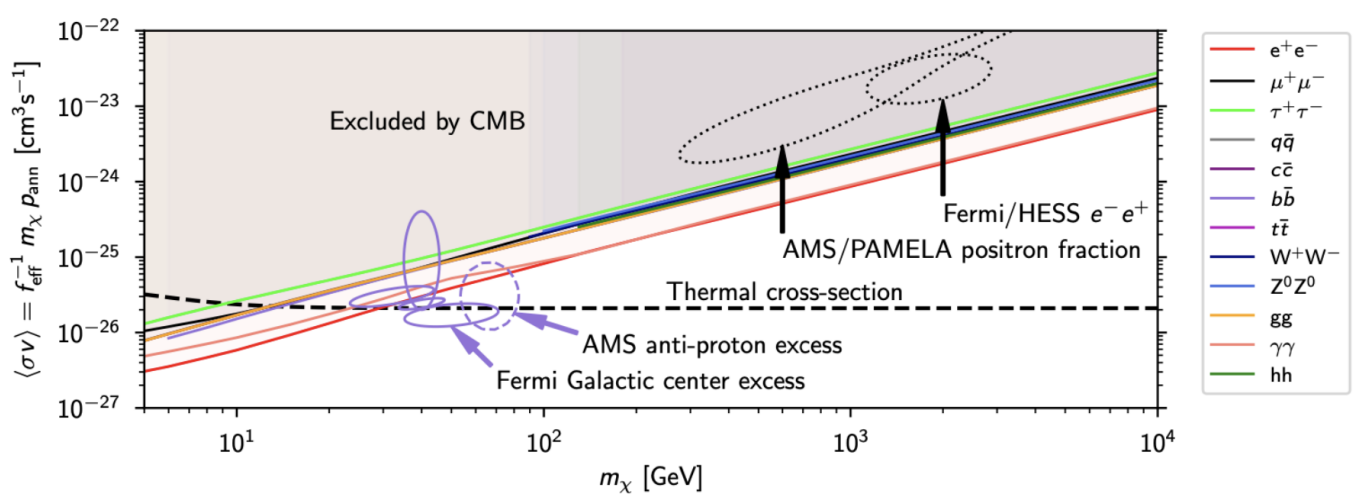

Figure 2. The CMB constraints on DM annihilation cross section. Different regions bounded by straight solid lines correspond to DM annihilation into different final states. Other regions are related to the observations of various particle fluxes as explained in more detail in the text. The figure is from [1].

\subsection{Portals between Dark and Light Sectors}

The previous sections have illustrated the general features of dark sectors on cosmology and emphasized the role of the mediators. In the case of direct coupling the mediator is essential for setting the relic abundance. The minimum coupling needed for the successful generation of the relic density then sets the expectations for the experiments, searching for the signal of dark matter interactions. In the isolated dark sector framework, an additional interaction between dark sector and SM is not needed to establish the relic abundance, 
but may assist in depleting the excess energy and entropy away from the dark sector. The existence of such interactions then opens up the possibilities to search for DM in laboratory experiments also in this type of model, although the possibilities for the signal strengths are more varied. Let us now briefly discuss the most commonly studied mediator portals with the SM.

\subsubsection{The Higgs Portal}

The most straightforward example is the Higgs portal, since the SM operator $H^{\dagger} H$ can couple with any suitable operator constructed out of the dark sector fields [128,129]. For example, a real scalar field $\phi$ in the dark sector will couple as $\phi^{2} H^{\dagger} H$ and $\phi H^{\dagger} H$. Due to these interactions, the dark sector scalar and the Higgs will mix after electroweak symmetry breaking. Hence, in the zero temperature vacuum, the interactions of the dark scalar with SM gauge and matter fields will arise. Simple models of DM in this context include hidden sectors with real scalar [128], complex scalar [130], fermion [131,132] or vector $[38,133]$ fields.

\subsubsection{The Vector Portal}

If there is a $\mathrm{U}(1)$ gauge field $V_{\mu}$ in the dark sector, it will mix with the hypercharge $B_{\mu}$ [134]. Denoting the field strengths of the dark gauge field and the SM hypercharge by $V_{\mu \nu}$ and $B_{\mu \ni}$, respectively, the operator $V_{\mu \nu} B^{\mu \nu}$ is allowed by both the dark and visible sector gauge invariance. The field $V_{\mu}$ is typically called a dark photon $[135,136]$ since, after electroweak symmetry breaking, there is the mixing $V_{\mu \nu} F^{\mu \nu}$.

Phenomenologically, the relevant interactions are

$$
\Delta \mathcal{L}=\frac{\epsilon}{2} V_{\mu v} F^{\mu v}+e A_{\mu} J_{\mathrm{em}}^{\mu}+e_{\chi} V_{\mu} J_{\mathrm{D}^{\prime}}^{\mu}
$$

where $J_{\mathrm{em}}^{\mu}$ and $J_{\mathrm{D}}^{\mu}$ are, respectively, the ordinary electromagnetic current and the dark vector current arising from the new gauge symmetry with gauge coupling $e_{\chi}$. The parameter $\epsilon$ measures the magnitude of the kinetic mixing. Both photons have also their corresponding kinetic terms and also any charged dark particles constituting the dark current have appropriate kinetic and mass terms. There is also the mass term for the vector $V_{\mu}, m_{V} / 2 V_{\mu} V^{\mu}$, which can arise from the dark Higgs mechanism. The simplest case is to assume that the dark Higgs-like scalar is massive and can be integrated out for the DM phenomenology, but it can also be considered a light degree of freedom to be included in the phenomenology analyses of the model.

The scenarios corresponding to $m_{V}=0$ and $m_{V} \neq 0$ need to be treated separately. Let us start with the case $m_{V}=0$. Then, the kinetic mixing can be eliminated by a field redefinition $V_{\mu}^{\prime}=V_{\mu}-\epsilon A_{\mu}$, which leaves us with the following interactions

$$
\Delta \mathcal{L}=e A_{\mu} J_{\mathrm{em}}^{\mu}+e_{\chi}\left(V_{\mu}^{\prime}+\epsilon A_{\mu}\right) J_{\mathrm{D}}^{\mu}
$$

If there are no charged fields in the dark sector, the vector $V_{\mu}^{\prime}$ would be completely decoupled and without observable effects. We therefore expect that in this limit the only observable consequences arise from additional matter fields in the dark sector. For example, suppose that $J_{\mathrm{D}}^{\mu}=\bar{\chi} \gamma^{\mu} \chi$. Then the DM field $\chi$ couples with the photon by the coupling $\epsilon e_{\chi}$. This provides an example of millicharged DM which is heavily constrained by its behavior in the early universe and resulting effects on structure formation [137] and CMB anisotropies [138].

In the case of $m_{v} \neq 0$, it is convenient to make a field redefinition $A_{\mu}^{\prime}=A_{\mu}-\epsilon V_{\mu}$ in order to again remove the kinetic mixing and to obtain

$$
\Delta \mathcal{L}=m_{V}^{2} / 2 V_{\mu} V^{\mu}+e\left(A_{\mu}^{\prime}+\kappa V_{\mu}\right) J_{\mathrm{EM}}^{\mu}+e_{\chi} V_{\mu} J_{\mathrm{D}}^{\mu}
$$

This is convenient as it immediately implies that the dark photon mass eigenstate $V_{\mu}$ couples to the particles charged under SM. The kinetic mixing parameter $\epsilon$ and the dark photon 
mass $m_{V}$ can be constrained over a wide range; for a recent compilation of constraints, see [139].

\subsubsection{Axion Portal}

An axion-like particle $a$ can couple with SM fermions or gauge bosons via operators

$$
\frac{1}{F_{a}} \partial_{\mu} a \bar{f} \gamma^{\mu} \gamma^{5} f, \quad \frac{1}{F_{a}} a F \tilde{F}, \quad \frac{1}{F_{a}} a G_{b, \mu \nu} \tilde{G}_{b}^{\mu v}
$$

where $F_{a}$ is the axion decay constant and $F_{\mu \nu}$ and $G_{\mu \nu}$ denote the electromagnetic and QCD field strengths, and the tilde their duals. Historically, the emergence of axion-like partilces is due to the Peccei and Quinn solution $[140,141]$ to the strong CP problem by promoting the CP-violating angle $\theta$ in the QCD sector of the SM to a pseudoscalar field whose potential sets its value to zero.

In various extensions of the SM such axion-like particles arise in connection with the strong CP problem or otherwise, and effectively the axion mass and decay constant are independent parameters. The models of this type fall in two classes of models: models where fermions dominate the axion couplings are of the type proposed by Dine, Fischler, Srednicki and Zhitnitsky $[142,143]$ while the models where the couplings to the gauge bosons are larger are of the type proposed by Kim, Shifman, Vainhstein and Zakharov, [144,145].

Axions in the sub-eV mass range are excellent DM candidates. They are produced non-thermally in the early universe via a misaligment mechanism [146,147]. However, being ultralight and outside the mass window of CDM, which is the focus of this review, we do not pursue these details further; see, e.g., [148].

\subsubsection{Neutrino Portal}

If sterile neutrinos $N_{i}$ exist, then there is also the interaction

$$
\Delta \mathcal{L}=y_{i j} \bar{L}_{i} H N_{j}
$$

where $L$ is a SM lepton doublet. This arises, for example, in the type I seesaw mechanism, which explains the smallness of neutrino masses in the SM by assuming heavy Majorana mass terms for the $N_{i}$. More general mass patterns for $N$ can be considered by embedding the above interaction into

$$
\Delta \mathcal{L}=y_{i j} \bar{L}_{i} H N_{j}+\frac{1}{2} M_{i j} N_{i} N_{j}+m_{i j} \bar{N}_{i} N_{j}
$$

Addition of the mass terms leads to more involved model building concerning their origin. In the simplest extension, one may add a singlet scalar with Yukawa couplings with the singlet fermions. Then, one needs to also take into account the Higgs portal interactions of the singlet scalar.

\section{Strongly Coupled Composite Dark Matter}

Particle dark matter possesses two main features: stability over timescales of the age of the universe and effective neutrality under electromagnetism. If we look at particles inside the standard model, there are two examples, each satisfying one of these constraints. First, there is the proton which, albeit charged, has an extremely long lifetime. Second, there is neutron which has zero net electric charge. Both of these particles are not elementary, but are composite objects whose internal dynamics is governed by the strongly coupled gauge theory, QCD. Generally, the explicit or accidental symmetries of gauge-fermion systems or scale dependent interactions, interpolating between different IR and UV behaviors, offer wide possibilities for dark matter model building.

These observations provide motivation for the investigation of dark sectors with nonAbelian gauge interactions, which allow for dark matter candidates as strongly-coupled composite states. Such composite dark sectors can arise in extensions of the SM, in which 
the Higgs sector itself is composite, or composite dark sectors can be assumed to exist on their own while the SM Higgs is described by the effective SM-like elementary scalar boson. Further motivation is provided by the strong self-interactions among the bound state of a strongly-coupled theory, which, as discussed in Section 3, may play a role in explaining features observed in formation of galactic structures.

One can model the composite dark sectors either using bottom up low energy effective theory. Then, when studying energy scales below the compositeness scale, the dark sector fields are treated as elementary but taking into account the series of irrelevant operators compatible with symmetries of the SM and the dark sector fields. The possible terms in the effective Lagrangian are constrained by general theoretical restrictions and observations. Alternatively, one can work within a top-down approach, starting with a specific ultraviolet completion to restrict the spectrum of operators generated at low energies. Lattice calculations provide an important tool to assess nonperturbative features of these models.

\subsection{General Features and Examples of Composite Dark Matter Candidates}

We will start with a brief review of general features and motivations of strongly coupled dark matter candidates. Then we will outline a few concrete example models and how they encapsulate the general features. Several dedicated reviews focusing on different aspects of composite dark matter exits in the literature and should be consulted for further details.

As already emphasized, the main feature of dark matter is its stability. In composite dark matter scenarios, this stability can arise naturally from accidental global flavor symmetries of the microscopic theory. In the SM, the stability of the proton arises in this manner due to the baryon number conservation. The symmetry violations arise as irrelevant operators, $\mathcal{O}_{d}$ with operator dimension $d$ determined by the underlying theory. If the operator dimension $d$ is large enough, then Planck suppressed operators, $\mathcal{O}_{d} / M_{\mathrm{P}}^{d-4}$, will allow for dark matter with the lifetime exceeding the age of the universe.

The other main feature of dark matter is its neutrality under electromagnetism and effectively under all SM charges. In composite dark matter models, this arises by the confinement which, analogously with QCD, arranges the physical spectrum of the theory in terms of dark hadrons. These spectra can contain states which are neutral under color, weak and electromagnetic, even if the fundamental constituents transform nontrivially under (some) of the SM symmetries. Dynamical confinement and breaking of the chiral symmetries in the dark sector leads to the intrinsic scale $\Lambda_{\mathrm{DM}}$ in the dark sector. This dark sector scale is, therefore, natural and may or needs not be related to the electroweak scale of the SM.

In comparison with the elementary fields, the interactions of the composite dark matter have notable differences. First, since composite DM is singlet with respect to the SM interactions, its interactions with SM fields appear at low energies as higher dimensional operators suppressed by the powers of the dark sector confinement scale. This suppresses the dark matter scattering off nuclei, which is relevant in the interpretation of the results in dark matter direct detection experiments. Second, the dark hadrons naturally have strong self-interactions which may play an important role in explaining features in the small scale structure formation, as we have discussed earlier. As a third point, we note that the spectrum of composite states within the dark sector provides significant refinements to DM scattering phenomena in comparison to simple dark sectors consisting of only few elementary fields. Examples include inelastic scattering [149], dark absorption [150] and effects on CMB and $N_{\text {eff }}$ [151].

Below, we enlist briefly some DM candidates arising in the setting of composite DM and strongly coupled hidden sectors. 


\subsubsection{Dark Pions}

Models of a QCD-like dark sector have been much discussed in the literature [152-155]. It is imagined to arise from the underlying strong dynamics with light dark quarks, $m_{q} \ll \Lambda_{\mathrm{DM}}$. We note here that although these models are interesting purely for DM phenomenology, they may also generate the electroweak scale starting from classically scale invariant Lagrangian [155]. Different models of this type have been studied recently and the dynamical symmetry breaking relevant for strongly coupled hidden sectors is only one possiblility while, e.g., radiative symmetry breaking is another alternative [156].

To illustrate these dynamics, consider the model where a dark strongly coupled nonabelian gauge theory is coupled with a SM-like elementary scalar boson and a messenger scalar, an elementary real singlet field $S$, which provides for a Higgs portal to the dark sector and transmits the dark sector scale to the electroweak sector of the SM [155]. More concretely, the relevant Lagrangian is

$$
\begin{aligned}
\mathcal{L} & =\left|D_{\mu} H\right|^{2}-\lambda_{h}\left(H^{\dagger} H\right)^{2}+\left|\partial_{\mu} S\right|^{2}-\lambda_{S}|S|^{4}-\frac{1}{4} F_{\mu \nu}^{a} F^{a \mu \nu} \\
& +\sum_{i=1}^{N_{f}} \bar{Q}_{i}\left(i \gamma^{\mu} D_{\mu}\right) Q_{i}+g_{h}|S|^{2}|H|^{2}-\left(y_{Q} S \bar{Q} Q+\text { h.c. }\right) .
\end{aligned}
$$

Here we have denoted the SM-like Higgs doublet by $H$ and the covariant derivative operating on it contains the appropriate SM gauge fields. The dark sector is here written in terms of the underlying elementary degrees of freedom. The dark gauge field strength is denoted by $F_{\mu \nu}^{a}$ and the dark quark fields by $Q$. They are taken to transform in the fundamental representation of the dark gauge group and the covariant derivative acting on the dark quarks contains the dark gauge field. The singlet scalar $S$ has renormalizable interactions with itself, the Higgs and the dark quarks.

In the limit of massless quarks, there is chiral symmetry which, for sufficiently small number of flavors, is expected to be dynamically broken by the formation of dark quark condensate. The symmetry breaking pattern depends on if the dark quarks transform in complex, pseudoreal or real representation of the gauge group. We assume here that the gauge group is such that the fundamental representation is complex and, therefore, discuss only the QCD-like behavior, i.e., $\mathrm{SU}\left(N_{f}\right) \times \mathrm{SU}\left(N_{f}\right) \rightarrow \mathrm{SU}\left(N_{f}\right)$.

Due to the coupling with the scalar sector, the Goldstone bosons associated with the above symmetry breaking pattern will become pseudo-Goldstones. In the case we consider, all these are $\bar{Q} Q$-states. There is a remnant flavor symmetry, which protects the lightest pseudo-Goldstone against decay and this, therefore, becomes a dark matter candidate. The consequences of the formation of the $\bar{Q} Q$-condensate and the associated scale $\Lambda_{\mathrm{DM}}$ are transmitted to the SM sector via the Higgs portal by formation of vacuum expectation values for both $S$ and $H$. In this way, the model also explains the origin of the SM masses and simultaneously ties them with the dark matter dynamics.

The low-energy phenomenology is most conveniently discussed in terms of a lowenergy effective theory where the relevant degrees of freedom are the composite Goldstone bosons $\pi$ and the scalar $\sigma$ parametrised in the linear representation as a multiplet $M \sim \sigma+i \pi \cdot \tau$. For details and further discussion of the phenomenology, see [155].

In the above example the dark sector fields are singlet under all SM interactions. Alternatively, one can also consider a model building framework where both DM and the SM Higgs emerge as composite states from the same strongly coupled sector [157]. However, in models of this type, to avoid the topological anomaly $[158,159]$ leading to DM decay, one must work with large global symmetries [160]. In relation to this, we note that the same symmetry-breaking patterns can also be investigated in a setting where both the Higgs and DM are elementary fields [161]. For a review on the differences between the composite and elementary fields in this framework, see [162].

Various other possibilities for SM charge assignments in the strongly coupled dark sector can of course be considered. For example, in [152], the dark quarks transform in a 
vector-like representation of dark $\mathrm{SU}(N)$ and weak $\mathrm{SU}(2)_{\mathrm{L}}$, while in [163] the dark quarks transform instead in a vector-like representation of $\mathrm{U}(1)_{\mathrm{Y}}$.

\subsubsection{Dark Quarkonia and Dark Baryons}

There is no need to assume that the fermion constituents of dark sector composite states would be light. For example, one can consider the regime where at least one dark fermion is heavy $m_{Q}>\Lambda_{\mathrm{DM}}$. Then, the effective theory to be applied is heavy quark effective theory and the dark matter candidate is an analogue of quarkonia. This possibility was considered, for example, in the model presented in [164], where one of the main phenomenological features is the inelastic scattering [165-167]. Of course these can be tuned in the models with elementary fields, but they occur naturally in the strongly coupled composite scenarios.

Removing the light-heavy splitting and taking all dark fermions to be heavier than the dynamical dark matter scale leads to so-called "quirky" dark matter [150]. In this scenario, the dark fermions transform in addition to the new confining non-Abelian group also under part of the SM [168]. Then, one must also take into account the constraints from vacuum alignment [169]. For a model analysis, see, e.g., [170].

For dark mesons in general, it may be difficult to avoid breaking the global flavor symmetries by irrelevant operators of dimension five. This then undermines the requirement of dark matter stability over the cosmologically relevant timescales. This situation motivates to study baryon-like DM candidates for which the stability is more robust. For example, in SU(3) and larger number of colors, the higher dimensional operators are at least of dimension six and DM remains sufficiently stable.

The work on technibaryons dates back to technicolor models [18,171,172]. In these models, the new fermions transformed under a chiral representation of the electroweak gauge group and via dynamical breaking of their chiral symmetries led to electroweak symmetry breaking. The technibaryon bound states carrying an accidental technibaryon number led to the suggestion of the lightest of these states as a DM candidate. Moreover, it turned out that the relic abundance of this type of DM could be obtained through an asymmetry intertwined with the generation of the ordinary matter-antimatter asymmetry [173,174].

\subsubsection{Dark Glueballs}

Generally, the spectrum of a non-abelian gauge theory contains bound states with no valence quark content, i.e., glueballs. In the case that all dark fermions have masses far above $\Lambda_{\mathrm{DM}}$, the lightest particles in the spectrum will be glueballs with masses around $\Lambda_{\mathrm{DM}}$ Being the lightest particles, their stabilization is endangered only by possible couplings with SM fields. The leading operators to couple dark glueballs with the SM fields are

$$
\mathcal{L} \sim \frac{c_{1}}{\Lambda^{2}} H^{\dagger} H \operatorname{Tr}\left(G_{\mu \nu} G^{\mu \nu}\right)+\frac{c_{2}}{\Lambda^{4}} \operatorname{Tr}\left(G_{\mu \nu} G^{\mu \nu}\right) \operatorname{Tr}\left(F_{\mu v} F^{\mu \nu}\right),
$$

where $G_{\mu \nu}$ is the field strength of the dark gauge field and $F_{\mu \nu}$ is the field strength of a SM gauge field and $H$ is the SM Higgs doublet. The scale $\Lambda$ is related with the new physics carrying both SM and dark quantum numbers and which is integrated out below the scale $\Lambda$ to generate the above effective interactions below scale $\Lambda$.

For example the Higgs coupling in the above equation leads to the decay rate $\Gamma_{G} \sim c_{1} \Lambda_{\mathrm{DM}}^{9} /\left(m_{H}^{4} \Lambda^{4}\right)$. If $\Lambda \mathrm{DM}$ is sufficiently small with respect to the new physics scale $\Lambda^{4}$, the glueballs can be stabilized with respect to the lifetime of the universe [175].

\section{Experimental and Observational Constraints}

Already in the earlier sections, we have described some experimental and observational constraints on different models of CDM. In this section, we will outline some more features and how different observational probes intertwine to constrain the models. 


\subsection{Collider Searches}

For the collider searches to be relevant, the situation is similar to direct detection: DM must interact sufficiently strongly with ordinary matter fields to yield observable signatures.

There are intensive searches for WIMP DM at colliders. For scalar DM coupled with the SM via the Higgs portal, the production of DM states is impeded by a small cross section [176] but searches via monojet events in Higgs decays are more promising [177] although the kinematic reach is limited. For collider constraints on fermion DM in a Higgs portal model, see [176]. Additionally, the freeze-in scenario could result in collider signals. For example, the appearance of particles with macroscopic lifetimes $[178,179]$ may arise due to the almost-isolated dark sector. For searches for long-lived particles, see, e.g., $[180,181]$. It is also possible that the dark sector where the DM abundance is generated by the freeze-in mechanism also contains a mediator particle coupling more strongly to the SM. This mediator could lead to observables that are relevant to collider experiments [179,182-184].

In Higgs portal models, there are two important bounds arising from the current collider experiments. First, if there are scalar states mixing with the Higgs, the measured Higgs couplings constrain the mixing angle. The current bound is $\sin \alpha \lesssim 0.3$ [185]. Furthermore, if DM is sufficiently light with respect to the Higgs boson, $m_{\chi}<m_{h^{0}} / 2$, the invisible Higgs decays provide an important bound. Currently, such decay is bounded by $\operatorname{Br}\left(h^{0} \rightarrow\right.$ inv $) \leq 0.23[186,187]$.

In composite DM scenarios, the collider signatures are affected by extended spectrum of state and by nontrivial form factors. For a review of different effects and an outline of different models, see [19] and references therein.

\subsection{Direct and Indirect Detection}

According to the CDM paradigm, a flux of DM particles bound to the galactic halo is constantly passing through the Earth. If coupled with ordinary matter, these particles would occasionally scatter on targets of ordinary matter and deposit a small amount of recoil energy. The direct detection experiments aim to measure these small energy deposits. Most of the past and current experiments have their focus on DM scattering on nuclei. In the past, there were traces of a DM signal, such as DAMA/LIBRA [188,189], CoGent [190-194] and CDMS-II [195]. Assuming a vanilla-type DM scattering, these observations are inconsistent with most recent experimental results [196,197]

The direct detection provides very stringent constraints for frozen-out WIMP DM candidates. The simple Higgs portal models are practically ruled out except for a narrow window close to the Higgs resonance [198]. For a comparison of Higgs portal models, taking into account existing constraints, see [199].

It is possible to avoid the constraints from direct detection while simultaneously maintaining large enough annihilation cross section so that the observed abundance is obtained if the energy dependence of the DM interactions is nontrivial. A concrete example of this occurs if the DM particle is a pseudo-Goldstone boson [130,200]. Goldstone bosons generally scatter with cross sections proportional to the momentum transfer in the process. This dependency leads to the low energy DM scattering cross section on ordinary matter which are heavily suppressed. For example consider the model [130] defined by a Lagrangian

$$
\mathcal{L}=\frac{1}{2}\left(\partial_{\mu} S\right)^{2}-V(S, H)
$$

where $S=\left(\eta_{1}, \eta_{2}, \ldots, \eta_{N-1}, \sigma\right)$ is an $\mathrm{O}(N)$ vector carrying no SM quantum numbers, $H$ is the SM-Higgs field and $V(H, S)$ is the potential invariant under $\mathrm{O}(N)$ transformations and SM gauge symmetries and containing also the leading symmetry breaking term $M_{\eta}^{2} \eta_{a} \eta_{a}$. 
The simplest model corresponds to $N=2$ where $S=\sigma+i \eta$. In this model, one finds that the cross section for DM scattering on ordinary matter is

$$
\frac{d \sigma_{\mathrm{SI}}}{d \cos \theta} \sim \frac{\lambda_{\mathrm{HS}}^{2} f_{N} m_{N}^{2}}{\left(m_{h^{0}}^{2}-t\right)^{2}\left(m_{H_{0}}^{2}-t\right)^{2}} t^{2}
$$

where $\lambda_{\mathrm{HS}}$ is the Higgs portal coupling, $h^{0}$ and $H^{0}$ are the two massive scalars with $h^{0}$ identified as the SM Higgs boson and $f_{N} \simeq 0.3$ [201] is the Higgs nucleon coupling. Hence, the direct detection cross section vanishes as $t \rightarrow 0$ appearing to resolve the tension with experimental constraints.

However, there is an important refinement: the symmetry breaking contributions, such as a small mass of the Goldstone boson, do not vanish in proportion to the momentum transfer. Consequently, symmetry breaking contributions can become significant effect even if generated at one-loop or even higher orders [202]. The quantitive effect is illustrated in Figure 3 adapted from [130]. In the left panel of the figure are shown the limits from XENON1T on the spin-independent scattering of DM off of nuclei assuming the leading order momentum-suppression due to the Goldstone-nature of DM as the upper shaded region. The shaded region below this corresponds to the domain where the SI cross section reaches the coherent neutrino scattering cross section. The constraint from the invisible Higgs decays are shown by the shaded region on the left. The solid (dashed) curve shows the parameter values where the correct relic density is obtained for $m_{H^{0}}=500 \mathrm{GeV}$ $\left(m_{H^{0}}=750 \mathrm{GeV}\right)$. The yellow region is related to the explicit symmetry breaking terms and is explained in more detail in [130]. The right panel of Figure 3 corresponds to the left one, but contains the dominant one-loop contributions for the DM-nucleon cross section and for the $m_{H^{0}}=500 \mathrm{GeV}$.
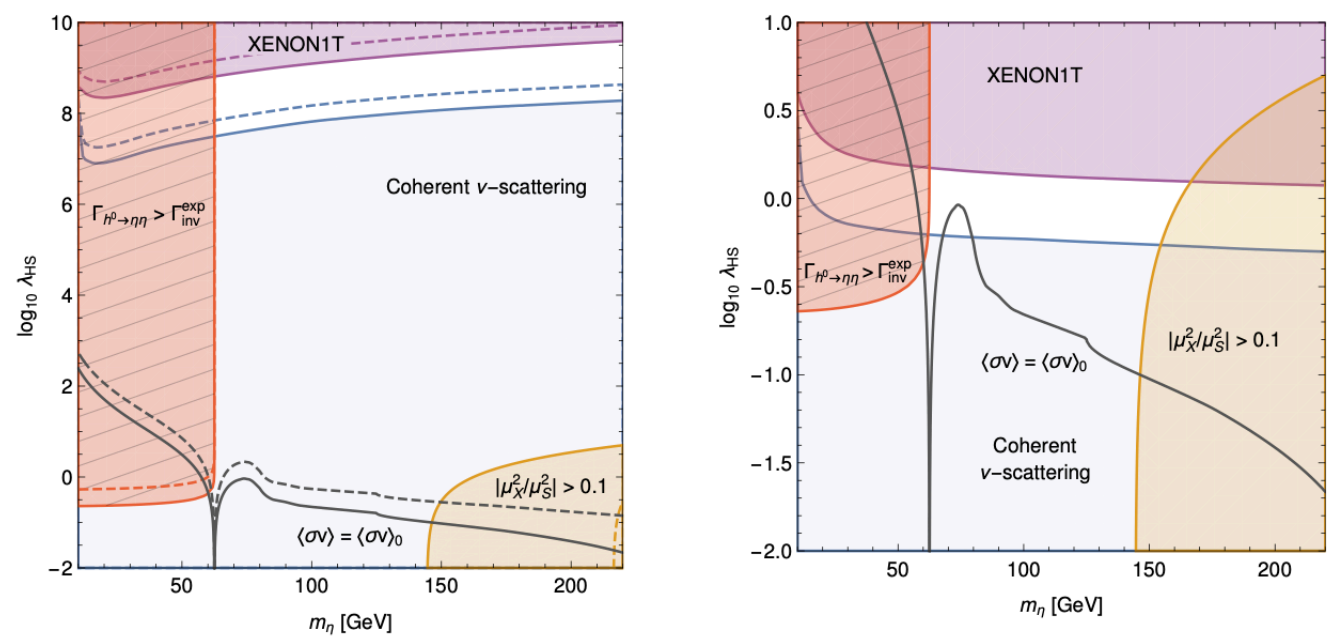

Figure 3. Illustration of different constraints on the DM-nucleon scattering. The horizontally separated shaded regions show the exclusion limits from the XENON1T direct detection experiment and from the saturation of the coherent neutrino scattering cross section while the shaded region in the left corresponds to the bound from the invisible Higgs decays. The solid (dashed) line corresponds to the parameter values where the correct DM relic density is obtained for $m_{H^{0}}=500 \mathrm{GeV}$ $\left(m_{H^{0}}=750 \mathrm{GeV}\right)$. The left panel shows the situation for the leading order scattering cross section while the left panel shows the quantitiative change when the dominant one-loop contributions are also taken into account. Figure from [130].

Generally direct detection experiments are complemented with numerous indirect probes, which are particularly important for dark matter scenarios directly coupled with the $\mathrm{SM}$ as we have discussed in earlier sections for frozen-out DM. Indirect DM searches probe the same annihilation process that is relevant for determining the abundance, and for the pseudo-Goldstone boson DM it is particularly important that this scattering amplitude 
does not vanish in the limit of zero incoming three momentum. Therefore, the indirect detection signal is expected to be of similar magnitude for pseudo-Goldstone boson DM as for a generic WIMP. It turns out that indirect detection is very constraining for such DM candidates up to few hundred GeV [130].

Of the various other models we have considered, the FIMP paradigm easily escapes direct detection experiments due to extremely weak couplings between the dark and visible sectors. However, they can accommodate interesting indirect detection signals since the feeble couplings can result in very long lifetimes leading to decaying DM scenarios. For example, for a decaying DM interpretation of the spectral feature at $E \simeq 3.55 \mathrm{keV}[203,204]$, see [205-211].

Models with extended scalar sectors or strong dynamics with dynamical chiral symmtery breaking induce additional phase transitions in the early universe. If these are of first order and sufficiently strong, they can yield a stochastic gravitational wave signal detectable by future satellites BBO [212] and DECIGO [213]. In the case of pseudoGoldstone DM this feature also increases the direct detection signal [200].

\section{Conclusions}

We have considered models for CDM, i.e., particle dark matter whose mass is above the $\mathrm{keV}$ scale. We reviewed, in particular, models constituted by hidden sectors coupled with the SM fields only with a restricted set of mediator fields. Such a framework is mainly motivated by the observation of the SM-like Higgs boson at the LHC and no extended spectrum of beyond SM states.

We considered the production mechanisms of CDM in the early universe by thermal decoupling, i.e., freeze-out from the thermal SM heat bath or by out of equilibrium production by population of phase space via decays or scatterings of the bath particles, i.e., the freeze-in. We also discussed extensions of these two basic scenarios, dark freeze-out and reannihilation. Alternatives to these mechanisms are asymmetric dark matter. Asymmetric dark matter arises naturally in the composite dark matter scenarios which are also motivated by dark matter self-interactions.

The observational constraints were discussed briefly, and new developments in direct detection and ongoing developments in the studies of astrophysical probes of indirect DM detection are expected to further constrain the CDM paradigm.

Funding: This research was funded by the ACADEMY OF FINLAND grant number 310130.

Conflicts of Interest: The author declares no conflict of interest.

\section{References}

1. Aghanim, N.; Akrami, Y.; Ashdown, M.; Aumont, J.; Baccigalupi, C.; Ballardini, M.; Banday, A.J.; Barreiro, R.B.; Bartolo, N.; Basak, S.; et al. Planck 2018 results. VI. Cosmological parameters. Astron. Astrophys. 2020, 641, A6; Erratum in 2021, 652, C4. [CrossRef]

2. Bergström, L. Nonbaryonic dark matter: Observational evidence and detection methods. Rep. Prog. Phys. 2000, 63, 793. [CrossRef]

3. Bertone, G.; Hooper, D. History of dark matter. Rev. Mod. Phys. 2018, 90, 045002. [CrossRef]

4. de Swart, J.; Bertone, G.; van Dongen, J. How Dark Matter Came to Matter. Nat. Astron. 2017, 1, 0059. [CrossRef]

5. Kawasaki, M.; Kohri, K.; Sugiyama, N. MeV scale reheating temperature and thermalization of neutrino background. Phys. Rev. D 2000, 62, 023506. [CrossRef]

6. Hannestad, S. What is the lowest possible reheating temperature? Phys. Rev. D 2004, 70, 043506. [CrossRef]

7. Ichikawa, K.; Kawasaki, M.; Takahashi, F. The Oscillation effects on thermalization of the neutrinos in the Universe with low reheating temperature. Phys. Rev. D 2005, 72, 043522. [CrossRef]

8. De Bernardis, F.; Pagano, L.; Melchiorri, A. New constraints on the reheating temperature of the universe after WMAP-5. Astropart. Phys. 2008, 30, 192-195. [CrossRef]

9. Hui, L.; Ostriker, J.P.; Tremaine, S.; Witten, E. Ultralight scalars as cosmological dark matter. Phys. Rev. D 2017, 95, 043541. [CrossRef]

10. Carr, B.; Kuhnel, F. Primordial Black Holes as Dark Matter: Recent Developments. Ann. Rev. Nucl. Part. Sci. 2020, 70, 355-394. [CrossRef]

11. Milgrom, M. A Modification of the Newtonian dynamics as a possible alternative to the hidden mass hypothesis. Astrophys. J. 1983, 270, 365-370. [CrossRef] 
12. Bekenstein, J.D. Relativistic gravitation theory for the MOND paradigm. Phys. Rev. D 2004, 70, 083509; Erratum in 2005, 71, 069901. [CrossRef]

13. Skordis, C.; Mota, D.F.; Ferreira, P.G.; Boehm, C. Large Scale Structure in Bekenstein's theory of relativistic Modified Newtonian Dynamics. Phys. Rev. Lett. 2006, 96, 011301. [CrossRef]

14. Zuntz, J.; Zlosnik, T.G.; Bourliot, F.; Ferreira, P.G.; Starkman, G.D. Vector field models of modified gravity and the dark sector. Phys. Rev. D 2010, 81, 104015. [CrossRef]

15. Lisanti, M.; Moschella, M.; Outmezguine, N.J.; Slone, O. Testing Dark Matter and Modifications to Gravity using Local Milky Way Observables. Phys. Rev. D 2019, 100, 083009. [CrossRef]

16. Arcadi, G.; Dutra, M.; Ghosh, P.; Lindner, M.; Mambrini, Y.; Pierre, M.; Profumo, S.; Queiroz, F.S. The waning of the WIMP? A review of models, searches, and constraints. Eur. Phys. J. C 2018, 78, 203. [CrossRef]

17. Bernal, N.; Heikinheimo, M.; Tenkanen, T.; Tuominen, K.; Vaskonen, V. The Dawn of FIMP Dark Matter: A Review of Models and Constraints. Int. J. Mod. Phys. A 2017, 32, 1730023. [CrossRef]

18. Nussinov, S. Technocosmology: Could a technibaryon excess provide a 'natural' missing mass candidate? Phys. Lett. B 1985, 165, 55-58. [CrossRef]

19. Kribs, G.D.; Neil, E.T. Review of strongly-coupled composite dark matter models and lattice simulations. Int. J. Mod. Phys. A 2016, 31, 1643004. [CrossRef]

20. Heikinheimo, M.; Tuominen, K.; Langæble, K. Hidden strongly interacting massive particles. Phys. Rev. D 2018, 97, 095040. [CrossRef]

21. Boucenna, S.M.; Krauss, M.B.; Nardi, E. Minimal Asymmetric Dark Matter. Phys. Lett. B 2015, 748, 191-198. [CrossRef]

22. Aad, G.; Abajyan, T.; AbbottJ, B.; Abdallah, J.; Abdel Khalek, S.; Abdelalim, A.A.; Abdinov, O.; Aben, R.; Abi, B.; Abolins, M.; et al. Observation of a new particle in the search for the Standard Model Higgs boson with the ATLAS detector at the LHC. Phys. Lett. B 2012, 716, 1-29. [CrossRef]

23. Chatrchyan, S.; Khachatryan, V.; Sirunyan, A.M.; Tumasyan, A.; Adam, W.; Aguilo, E.; Bergauer, T.; Dragicevic, M.; Erö, J.; Fabjan, C.; et al. Observation of a New Boson at a Mass of $125 \mathrm{GeV}$ with the CMS Experiment at the LHC. Phys. Lett. B 2012, 716, 30-61. [CrossRef]

24. Clowe, D.; Gonzalez, A.; Markevitch, M. Weak lensing mass reconstruction of the interacting cluster 1E0657-558: Direct evidence for the existence of dark matter. Astrophys. J. 2004, 604, 596-603. [CrossRef]

25. Kraljic, D.; Sarkar, S. How rare is the Bullet Cluster (in a $\Lambda$ CDM universe)? J. Cosmol. Astropart. Phys. 2015, 04, 050. [CrossRef]

26. Randall, S.W.; Markevitch, M.; Clowe, D.; Gonzalez, A.H.; Bradac, M. Constraints on the Self-Interaction Cross-Section of Dark Matter from Numerical Simulations of the Merging Galaxy Cluster 1E 0657-56. Astrophys. J. 2008, 679, 1173-1180. [CrossRef]

27. Heikinheimo, M.; Tenkanen, T.; Tuominen, K.; Vaskonen, V. Observational Constraints on Decoupled Hidden Sectors. Phys. Rev. D 2016, 94, 063506; Erratum in 2017, 96, 109902. [CrossRef]

28. McDonald, J. Thermally generated gauge singlet scalars as selfinteracting dark matter. Phys. Rev. Lett. 2002, 88, 091304. [CrossRef]

29. Hall, L.J.; Jedamzik, K.; March-Russell, J.; West, S.M. Freeze-In Production of FIMP Dark Matter. J. High Energy Phys. 2010, 03, 080. [CrossRef]

30. Kolb, E.W.; Turner, M.S. The Early Universe. Front. Phys. 1990, 69, 1-547.

31. McDonald, J. Gauge singlet scalars as cold dark matter. Phys. Rev. D 1994, 50, 3637-3649. [CrossRef]

32. Burgess, C.P.; Pospelov, M.; ter Veldhuis, T. The Minimal model of nonbaryonic dark matter: A Singlet scalar. Nucl. Phys. B 2001, 619, 709-728. [CrossRef]

33. Cline, J.M.; Kainulainen, K. Electroweak baryogenesis and dark matter from a singlet Higgs. J. Cosmol. Astropart. Phys. 2013, 01, 012. [CrossRef]

34. Zeldovic, Y.B.; Okun, L.B.; Pikelner, S.B. Quarks, astrophysical and physico-chemical aspects. Phys. Lett. 1965, 17, 164-166. [CrossRef]

35. Lee, B.W.; Weinberg, S. Cosmological Lower Bound on Heavy Neutrino Masses. Phys. Rev. Lett. 1977, 39, 165-168. [CrossRef]

36. Gondolo, P.; Gelmini, G. Cosmic abundances of stable particles: Improved analysis. Nucl. Phys. B 1991, 360, 145-179. [CrossRef]

37. Griest, K.; Seckel, D. Three exceptions in the calculation of relic abundances. Phys. Rev. D 1991, 43, 3191-3203. [CrossRef] [PubMed]

38. Hambye, T. Hidden vector dark matter. J. High Energy Phys. 2009, 01, 028. [CrossRef]

39. D'Agnolo, R.T.; Pappadopulo, D.; Ruderman, J.T. Fourth Exception in the Calculation of Relic Abundances. Phys. Rev. Lett. 2017, 119, 061102. [CrossRef]

40. Enqvist, K.; Nurmi, S.; Tenkanen, T.; Tuominen, K. Standard Model with a real singlet scalar and inflation. J. Cosmol. Astropart. Phys. 2014, 08, 035. [CrossRef]

41. Petraki, K.; Husenko, A. Dark-matter sterile neutrinos in models with a gauge singlet in the Higgs sector. Phys. Rev. D 2008, 77, 065014. [CrossRef]

42. Dev, P.S.B.; Mazumdar, A.; Qutub, S. Connection between dark matter abundance and primordial tensor perturbations. arXiv 2014, arXiv:1412.3041.

43. Nurmi, S.; Tenkanen, T.; Tuominen, K. Inflationary Imprints on Dark Matter. J. Cosmol. Astropart. Phys. 2015, 11, 001. [CrossRef]

44. Kainulainen, K.; Nurmi, S.; Tenkanen, T.; Tuominen, K.; Vaskonen, V. Isocurvature Constraints on Portal Couplings. J. Cosmol. Astropart. Phys. 2016, 06, 022. [CrossRef] 
45. Choi, K.Y.; Roszkowski, L. E-WIMPs. AIP Conf. Proc. 2005, 805, 30-36. [CrossRef]

46. Yaguna, C.E. An intermediate framework between WIMP, FIMP, and EWIP dark matter. J. Cosmol. Astropart. Phys. 2012, $02,006$. [CrossRef]

47. Krauss, M.B.; Morisi, S.; Porod, W.; Winter, W. Higher Dimensional Effective Operators for Direct Dark Matter Detection. J. High Energy Phys. 2014, 02, 056. [CrossRef]

48. Dolgov, A.D. On concentration of relict theta particles. Yad. Fiz. 1980, 31, 1522-1528. (In Russian)

49. Carlson, E.D.; Machacek, M.E.; Hall, L.J. Self-interacting dark matter. Astrophys. J. 1992, 398, 43-52. [CrossRef]

50. Chu, X.; Hambye, T.; Tytgat, M.H.G. The Four Basic Ways of Creating Dark Matter Through a Portal. J. Cosmol. Astropart. Phys. 2012, 05, 034. [CrossRef]

51. Bernal, N.; Chu, X.; Pradler, J. Simply split strongly interacting massive particles. Phys. Rev. D 2017, 95, 115023. [CrossRef]

52. Williams, A.J.; Boehm, C.; West, S.M.; Albornoz Vasquez, D. Regenerating WIMPs in the Light of Direct and Indirect Detection. Phys. Rev. D 2012, 86, 055018. [CrossRef]

53. Bernal, N.; Chu, X.; Garcia-Cely, C.; Hambye, T.; Zaldivar, B. Production Regimes for Self-Interacting Dark Matter. J. Cosmol. Astropart. Phys. 2016, 03, 018. [CrossRef]

54. Bernal, N.; Chu, X. $\mathbb{Z}_{2}$ SIMP Dark Matter. J. Cosmol. Astropart. Phys. 2016, 01, 006. [CrossRef]

55. Cheung, C.; Elor, G.; Hall, L.J.; Kumar, P. Origins of Hidden Sector Dark Matter I: Cosmology. J. High Energy Phys. 2011, $03,042$. [CrossRef]

56. Cheung, C.; Elor, G.; Hall, L.J.; Kumar, P. Origins of Hidden Sector Dark Matter II: Collider Physics. J. High Energy Phys. 2011, 03, 085. [CrossRef]

57. Di Valentino, E.; Giusarma, E.; Lattanzi, M.; Mena, O.; Melchiorri, A.; Silk, J. Cosmological Axion and neutrino mass constraints from Planck 2015 temperature and polarization data. Phys. Lett. B 2016, 752, 182-185. [CrossRef]

58. Di Valentino, E.; Melchiorri, A.; Silk, J. Cosmological constraints in extended parameter space from the Planck 2018 Legacy release. J. Cosmol. Astropart. Phys. 2020, 01, 013. [CrossRef]

59. Archidiacono, M.; Calabrese, E.; Melchiorri, A. The Case for Dark Radiation. Phys. Rev. D 2011, 84, 123008. [CrossRef]

60. de Salas, P.F.; Pastor, S. Relic neutrino decoupling with flavour oscillations revisited. J. Cosmol. Astropart. Phys. 2016, $07,051$. [CrossRef]

61. Cyburt, R.H.; Fields, B.D.; Olive, K.A.; Yeh, T.H. Big Bang Nucleosynthesis: 2015. Rev. Mod. Phys. 2016, 88, 015004. [CrossRef]

62. Ackerman, L.; Buckley, M.R.; Carroll, S.M.; Kamionkowski, M. Dark Matter and Dark Radiation. Phys. Rev. D 2009, 79, 023519. [CrossRef]

63. Knapen, S.; Lin, T.; Zurek, K.M. Light Dark Matter: Models and Constraints. Phys. Rev. D 2017, 96, 115021. [CrossRef]

64. Cui, Y.; Huo, R. Visualizing Invisible Dark Matter Annihilation with the CMB and Matter Power Spectrum. Phys. Rev. D 2019, 100, 023004. [CrossRef]

65. Moore, B. Evidence against dissipationless dark matter from observations of galaxy haloes. Nature 1994, 370, 629. [CrossRef]

66. Flores, R.A.; Primack, J.R. Observational and theoretical constraints on singular dark matter halos. Astrophys. J. Lett. 1994, 427, L1-L4. [CrossRef]

67. Navarro, J.F.; Frenk, C.S.; White, S.D.M. A Universal density profile from hierarchical clustering. Astrophys. J. 1997, 490, 493-508. [CrossRef]

68. de Blok, W.J.G. The Core-Cusp Problem. Adv. Astron. 2010, 2010, 789293. [CrossRef]

69. Oh, S.H.; Brook, C.; Governato, F.; Brinks, E.; Mayer, L.; de Blok, W. J. G.; Brooks, A.; Walter, F. The central slope of dark matter cores in dwarf galaxies: Simulations vs. THINGS. Astron. J. 2011, 142, 24. [CrossRef]

70. Walker, M.G.; Penarrubia, J. A Method for Measuring (Slopes of) the Mass Profiles of Dwarf Spheroidal Galaxies. Astrophys. J. 2011, 742, 20. [CrossRef]

71. Boylan-Kolchin, M.; Bullock, J.S.; Kaplinghat, M. Too big to fail? The puzzling darkness of massive Milky Way subhaloes. Mon. Not. R. Astron. Soc. 2011, 415, L40. [CrossRef]

72. Garrison-Kimmel, S.; Boylan-Kolchin, M.; Bullock, J.S.; Kirby, E.N. Too Big to Fail in the Local Group. Mon. Not. R. Astron. Soc. 2014, 444, 222-236. [CrossRef]

73. Mac Low, M.M.; Ferrara, A. Starburst-driven mass loss from dwarf galaxies: Efficiency and metal ejection. Astrophys. J. 1999, 513, 142. [CrossRef]

74. Governato, F.; Brook, C.; Mayer, L.; Brooks, A.; Rhee, G.; Wadsley, J.; Jonsson, P.; Willman, B.; Stinson, G.; Quinn, T.; et al. At the heart of the matter: The origin of bulgeless dwarf galaxies and Dark Matter cores. Nature 2010, 463, 203-206. [CrossRef]

75. Silk, J.; Nusser, A. The massive black hole-velocity dispersion relation and the halo baryon fraction: A case for positive AGN feedback. Astrophys. J. 2010, 725, 556-560. [CrossRef]

76. Vera-Ciro, C.A.; Helmi, A.; Starkenburg, E.; Breddels, M.A. Not too big, not too small: The dark halos of the dwarf spheroidals in the Milky Way. Mon. Not. R. Astron. Soc. 2013, 428, 1696. [CrossRef]

77. Sawala, T.; Frenk, C.S.; Fattahi, A.; Navarro, J.F.; Bower, R.G.; Crain, R.A.; Vecchia, C.D.; Furlong, M.; Helly, J.C.; Jenkins, A.; et al. The APOSTLE simulations: Solutions to the Local Group's cosmic puzzles. Mon. Not. R. Astron. Soc. 2016, 457, 1931-1943. [CrossRef]

78. Fattahi, A.; Navarro, J.F.; Sawala, T.; Frenk, C.S.; Sales, L.V.; Oman, K.; Schaller, M.; Wang, J. The cold dark matter content of Galactic dwarf spheroidals: No cores, no failures, no problem. arXiv 2016, arXiv:1607.06479. 
79. Markevitch, M.; Gonzalez, A.H.; Clowe, D.; Vikhlinin, A.; David, L.; Forman, W.; Jones, C.; Murray, S.; Tucker, W. Direct constraints on the dark matter self-interaction cross-section from the merging galaxy cluster 1E0657-56. Astrophys. J. 2004, 606, 819-824. [CrossRef]

80. Harvey, D.; Massey, R.; Kitching, T.; Taylor, A.; Tittley, E. The non-gravitational interactions of dark matter in colliding galaxy clusters. Science 2015, 347, 1462-1465. [CrossRef]

81. Robertson, A.; Massey, R.; Eke, V. What does the Bullet Cluster tell us about self-interacting dark matter? Mon. Not. R. Astron. Soc. 2017, 465, 569-587. [CrossRef]

82. Kim, S.Y.; Peter, A.H.G.; Wittman, D. In the Wake of Dark Giants: New Signatures of Dark Matter Self Interactions in Equal Mass Mergers of Galaxy Clusters. Mon. Not. R. Astron. Soc. 2017, 469, 1414-1444. [CrossRef]

83. Robertson, A.; Massey, R.; Eke, V. Cosmic particle colliders: Simulations of self-interacting dark matter with anisotropic scattering. Mon. Not. R. Astron. Soc. 2017, 467, 4719-4730. [CrossRef]

84. Wittman, D.; Golovich, N.; Dawson, W.A. The Mismeasure of Mergers: Revised Limits on Self-interacting Dark Matter in Merging Galaxy Clusters. Astrophys. J. 2018, 869, 104. [CrossRef]

85. Massey, R.; Williams, L.; Smit, R.; Swinbank, M.; Kitching, T.D.; Harvey, D.; Jauzac, M.; Israel, H.; Clowe, D.; Edge, A.; et al. The behaviour of dark matter associated with four bright cluster galaxies in the $10 \mathrm{kpc}$ core of Abell 3827. Mon. Not. R. Astron. Soc. 2015, 449, 3393-3406. [CrossRef]

86. Kahlhoefer, F.; Schmidt-Hoberg, K.; Kummer, J.; Sarkar, S. On the interpretation of dark matter self-interactions in Abell 3827. Mon. Not. R. Astron. Soc. 2015, 452, L54-L58. [CrossRef]

87. Taylor, P.; Massey, R.; Jauzac, M.; Courbin, F.; Harvey, D.; Joseph, R.; Robertson, A. A test for skewed distributions of dark matter and a possible detection in galaxy cluster Abell 3827. Mon. Not. R. Astron. Soc. 2017, 468, 5004-5013. [CrossRef]

88. Tulin, S.; Yu, H.B.; Zurek, K.M. Beyond Collisionless Dark Matter: Particle Physics Dynamics for Dark Matter Halo Structure. Phys. Rev. D 2013, 87, 115007. [CrossRef]

89. Kaplinghat, M.; Tulin, S.; Yu, H.B. Direct Detection Portals for Self-interacting Dark Matter. Phys. Rev. D 2014, 89, 035009. [CrossRef]

90. Huo, R.; Kaplinghat, M.; Pan, Z.; Yu, H.B. Signatures of Self-Interacting Dark Matter in the Matter Power Spectrum and the CMB. Phys. Lett. B 2018, 783, 76-81. [CrossRef]

91. Egana-Ugrinovic, D.; Essig, R.; Gift, D.; LoVerde, M. The Cosmological Evolution of Self-interacting Dark Matter. J. Cosmol. Astropart. Phys. 2021, 05, 013. [CrossRef]

92. Boehm, C.; Dolan, M.J.; McCabe, C. A Lower Bound on the Mass of Cold Thermal Dark Matter from Planck. J. Cosmol. Astropart. Phys. 2013, 08, 041. [CrossRef]

93. Nollett, K.M.; Steigman, G. BBN And The CMB Constrain Light, Electromagnetically Coupled WIMPs. Phys. Rev. D 2014, 89, 083508. [CrossRef]

94. Slatyer, T.R.; Padmanabhan, N.; Finkbeiner, D.P. CMB Constraints on WIMP Annihilation: Energy Absorption During the Recombination Epoch. Phys. Rev. D 2009, 80, 043526. [CrossRef]

95. Goodenough, L.; Hooper, D. Possible Evidence For Dark Matter Annihilation In The Inner Milky Way From The Fermi Gamma Ray Space Telescope. arXiv 2009, arXiv:0910.2998.

96. Abazajian, K.N.; Kaplinghat, M. Detection of a Gamma-Ray Source in the Galactic Center Consistent with Extended Emission from Dark Matter Annihilation and Concentrated Astrophysical Emission. Phys. Rev. D 2012, 86, 083511; Erratum in 2013, 87, 129902. [CrossRef]

97. Daylan, T.; Finkbeiner, D.P.; Hooper, D.; Linden, T.; Portillo, S.K.N.; Rodd, N.L.; Slatyer, T.R. The characterization of the gamma-ray signal from the central Milky Way: A case for annihilating dark matter. Phys. Dark Univ. 2016, 12, 1-23. [CrossRef]

98. Zhou, B.; Liang, Y.F.; Huang, X.; Li, X.; Fan, Y.Z.; Feng, L.; Chang, J. GeV excess in the Milky Way: The role of diffuse galactic gamma-ray emission templates. Phys. Rev. D 2015, 91, 123010. [CrossRef]

99. Calore, F.; Cholis, I.; Weniger, C. Background Model Systematics for the Fermi GeV Excess. J. Cosmol. Astropart. Phys. 2015, 03, 038. [CrossRef]

100. Ajello, M.; Albert, A.; Atwood, W.B.; Barbiellini, G.; Bastieri, D.; Bechtol, K.; Bellazzini, R.; Bissaldi, E.; Blandford, R.D.; Bloom, E.D.; et al. Fermi-LAT Observations of High-Energy $\gamma$-Ray Emission Toward the Galactic Center. Astrophys. J. 2016, 819, 44. [CrossRef]

101. Linden, T.; Rodd, N.L.; Safdi, B.R.; Slatyer, T.R. High-energy tail of the Galactic Center gamma-ray excess. Phys. Rev. D 2016, 94, 103013. [CrossRef]

102. Petrović, J.; Serpico, P.D.; Zaharijaš, G. Galactic Center gamma-ray "excess" from an active past of the Galactic Centre? J. Cosmol. Astropart. Phys. 2014, 10, 052. [CrossRef]

103. Calore, F.; di Mauro, M.; Donato, F. Diffuse gamma-ray emission from galactic pulsars. Astrophys. J. 2014, 796, 1. [CrossRef]

104. Cholis, I.; Hooper, D.; Linden, T. Challenges in Explaining the Galactic Center Gamma-Ray Excess with Millisecond Pulsars. J. Cosmol. Astropart. Phys. 2015, 06, 043. [CrossRef]

105. Petrović, J.; Serpico, P.D.; Zaharijas, G. Millisecond pulsars and the Galactic Center gamma-ray excess: The importance of luminosity function and secondary emission. J. Cosmol. Astropart. Phys. 2015, 02, 023. [CrossRef]

106. Cholis, I.; Evoli, C.; Calore, F.; Linden, T.; Weniger, C.; Hooper, D. The Galactic Center GeV Excess from a Series of Leptonic Cosmic-Ray Outbursts. J. Cosmol. Astropart. Phys. 2015, 12, 005. [CrossRef] 
107. Macias, O.; Gordon, C.; Crocker, R.M.; Coleman, B.; Paterson, D.; Horiuchi, S.; Pohl, M. Galactic bulge preferred over dark matter for the Galactic centre gamma-ray excess. Nat. Astron. 2018, 2, 387-392. [CrossRef]

108. Calore, F.; Cholis, I.; McCabe, C.; Weniger, C. A Tale of Tails: Dark Matter Interpretations of the Fermi GeV Excess in Light of Background Model Systematics. Phys. Rev. D 2015, 91, 063003. [CrossRef]

109. Bertone, G.; Calore, F.; Caron, S.; Ruiz, R.; Kim, J.S.; Trotta, R.; Weniger, C. Global analysis of the pMSSM in light of the Fermi GeV excess: Prospects for the LHC Run-II and astroparticle experiments. J. Cosmol. Astropart. Phys. 2016, 04, 037. [CrossRef]

110. Albert, A.; Anderson, B.; Bechtol, K.; Drlica-Wagner, A.; Meyer, M.; Sánchez-Conde, M.; Strigari, L.; Wood, M.; Abbott, T.M.C.; Abdalla, F.B.; et al. Searching for Dark Matter Annihilation in Recently Discovered Milky Way Satellites with Fermi-LAT. Astrophys. J. 2017, 834, 110. [CrossRef]

111. Aguilar, M.; Cavasonza, L.A.; Alpat, B.; Ambrosi, G.; Arruda, L.; Attig, N.; Aupetit, S.; Azzarello, P.; Bachlechner, A.; Barao, F.; et al. Antiproton Flux, Antiproton-to-Proton Flux Ratio, and Properties of Elementary Particle Fluxes in Primary Cosmic Rays Measured with the Alpha Magnetic Spectrometer on the International Space Station. Phys. Rev. Lett. 2016, 117, 091103. [CrossRef]

112. Cui, M.Y.; Yuan, Q.; Tsai, Y.L.S.; Fan, Y.Z. Novel Dark Matter Constraints from Antiprotons in Light of AMS-02. Phys. Rev. Lett. 2017, 118, 191102. [CrossRef]

113. Cui, M.Y.; Yuan, Q.; Tsai, Y.L.S.; Fan, Y.Z. Possible dark matter annihilation signal in the AMS-02 antiproton data. Phys. Rev. Lett. 2017, 118, 191101. [CrossRef] [PubMed]

114. Huang, X.J.; Wei, C.C.; Wu, Y.L.; Zhang, W.H.; Zhou, Y.F. Antiprotons from dark matter annihilation through light mediators and a possible excess in AMS-02 $\bar{p} / p$ data. Phys. Rev. D 2017, 95, 063021. [CrossRef]

115. Li, T. Simplified dark matter models in the light of AMS-02 antiproton data. J. High Energy Phys. 2017, 04, 112. [CrossRef]

116. Feng, J.; Zhang, H.H. Dark Matter Search in Space: Combined Analysis of Cosmic Ray Antiproton-to-Proton Flux Ratio and Positron Flux Measured by AMS-02. Astrophys. J. 2018, 858, 116. [CrossRef]

117. Jin, H.B.; Wu, Y.L.; Zhou, Y.F. Astrophysical background and dark matter implication based on latest AMS-02 data. Astrophys. J. 2020, 901, 80. [CrossRef]

118. Jia, L.B. Interpretation of the gamma-ray excess and AMS-02 antiprotons: Velocity dependent dark matter annihilations. Phys. Rev. D 2017, 96, 055009. [CrossRef]

119. Cuoco, A.; Heisig, J.; Korsmeier, M.; Krämer, M. Probing dark matter annihilation in the Galaxy with antiprotons and gamma rays. J. Cosmol. Astropart. Phys. 2017, 10, 053. [CrossRef]

120. di Mauro, M.; Winkler, M.W. Multimessenger constraints on the dark matter interpretation of the Fermi-LAT Galactic center excess. Phys. Rev. D 2021, 103, 123005. [CrossRef]

121. Giesen, G.; Boudaud, M.; Génolini, Y.; Poulin, V.; Cirelli, M.; Salati, P.; Serpico, P.D. AMS-02 antiprotons, at last! Secondary astrophysical component and immediate implications for Dark Matter. J. Cosmol. Astropart. Phys. 2015, 09, 023. [CrossRef]

122. Adriani, O.; Barbarino, G.C.; Bazilevskaya, G.A.; Bellotti, R.; Boezio, M.; Bogomolov, E.A.;Bonechi, L.; Bongi, M.; Bonvicini, V.; Bottai, S.; et al. An anomalous positron abundance in cosmic rays with energies 1.5-100 GeV. Nature 2009, 458, 607-609. [CrossRef] [PubMed]

123. Aguilar, M.; Alberti, G.; Alpat, B.; Alvino, A.; Ambrosi, G.; Andeen, K.; Anderhub, H.; Arruda, L.; Azzarello, P.; Bachlechner, A.; et al. First Result from the Alpha Magnetic Spectrometer on the International Space Station: Precision Measurement of the Positron Fraction in Primary Cosmic Rays of 0.5-350 GeV. Phys. Rev. Lett. 2013, 110, 141102. [CrossRef] [PubMed]

124. Hooper, D.; Blasi, P.; Serpico, P.D. Pulsars as the Sources of High Energy Cosmic Ray Positrons. J. Cosmol. Astropart. Phys. 2009, 01, 025. [CrossRef]

125. di Mauro, M.; Donato, F.; Fornengo, N.; Lineros, R.; Vittino, A. Interpretation of AMS-02 electrons and positrons data. J. Cosmol. Astropart. Phys. 2014, 04, 006. [CrossRef]

126. Bai, Y.; Carena, M.; Lykken, J. The PAMELA excess from neutralino annihilation in the NMSSM. Phys. Rev. D 2009, 80, 055004. [CrossRef]

127. Chen, C.H.; Chiang, C.W.; Nomura, T. Dark matter for excess of AMS-02 positrons and antiprotons. Phys. Lett. B 2015, 747, 495-499. [CrossRef]

128. Silveira, V.; Zee, A. SCALAR PHANTOMS. Phys. Lett. B 1985, 161, 136-140. [CrossRef]

129. Patt, B.; Wilczek, F. Higgs-field portal into hidden sectors. arXiv 2006, arXiv:hep-ph/0605188.

130. Alanne, T.; Heikinheimo, M.; Keus, V.; Koivunen, N.; Tuominen, K. Direct and indirect probes of Goldstone dark matter. Phys. Rev. D 2019, 99, 075028. [CrossRef]

131. Kim, Y.G.; Lee, K.Y. The Minimal model of fermionic dark matter. Phys. Rev. D 2007, 75, 115012. [CrossRef]

132. Kim, Y.G.; Lee, K.Y.; Shin, S. Singlet fermionic dark matter. J. High Energy Phys. 2008, 05, 100. [CrossRef]

133. Hambye, T.; Tytgat, M.H.G. Confined hidden vector dark matter. Phys. Lett. B 2010, 683, 39-41. [CrossRef]

134. Holdom, B. Two U(1)'s and Epsilon Charge Shifts. Phys. Lett. B 1986, 166, 196-198. [CrossRef]

135. Jaeckel, J. A force beyond the Standard Model - Status of the quest for hidden photons. Frascati Phys. Ser. 2012, 56, 172-192.

136. Fabbrichesi, M.; Gabrielli, E.; Lanfranchi, G. The Dark Photon; Springer: Berlin/Heidelberg, Germany, 2021. [CrossRef]

137. McDermott, S.D.; Yu, H.B.; Zurek, K.M. Turning off the Lights: How Dark is Dark Matter? Phys. Rev. D 2011, 83, 063509. [CrossRef]

138. Dvorkin, C.; Blum, K.; Kamionkowski, M. Constraining Dark Matter-Baryon Scattering with Linear Cosmology. Phys. Rev. D 2014, 89, 023519. [CrossRef] 
139. Caputo, A.; Millar, A.J.; O’Hare, C.A.J.; Vitagliano, E. Dark photon limits: A cookbook. arXiv 2021, arXiv:2105.04565.

140. Peccei, R.D.; Quinn, H.R. CP Conservation in the Presence of Instantons. Phys. Rev. Lett. 1977, 38, 1440-1443. [CrossRef]

141. Peccei, R.D.; Quinn, H.R. Constraints Imposed by CP Conservation in the Presence of Instantons. Phys. Rev. D 1977, 16, 1791-1797. [CrossRef]

142. Dine, M.; Fischler, W.; Srednicki, M. A Simple Solution to the Strong CP Problem with a Harmless Axion. Phys. Lett. B 1981, 104, 199-202. [CrossRef]

143. Zhitnitsky, A.R. On Possible Suppression of the Axion Hadron Interactions. Sov. J. Nucl. Phys. 1980, 31, 260. (In Russian)

144. Kim, J.E. Weak Interaction Singlet and Strong CP Invariance. Phys. Rev. Lett. 1979, 43, 103. [CrossRef]

145. Shifman, M.A.; Vainshtein, A.I.; Zakharov, V.I. Can Confinement Ensure Natural CP Invariance of Strong Interactions? Nucl. Phys. B 1980, 166, 493-506. [CrossRef]

146. Preskill, J.; Wise, M.B.; Wilczek, F. Cosmology of the Invisible Axion. Phys. Lett. B 1983, 120, 127-132. [CrossRef]

147. Abbott, L.F.; Sikivie, P. A Cosmological Bound on the Invisible Axion. Phys. Lett. B 1983, 120, 133-136. [CrossRef]

148. Choi, K.; Im, S.H.; Shin, C.S. Recent progress in physics of axions or axion-like particles. Annu. Rev. Nucl. Part. Sci. 2021. [CrossRef]

149. Alves, D.S.M.; Behbahani, S.R.; Schuster, P.; Wacker, J.G. The Cosmology of Composite Inelastic Dark Matter. J. High Energy Phys. 2010, 06, 113. [CrossRef]

150. Kribs, G.D.; Roy, T.S.; Terning, J.; Zurek, K.M. Quirky Composite Dark Matter. Phys. Rev. D 2010, 81, 095001. [CrossRef]

151. Garcia Garcia, I.; Lasenby, R.; March-Russell, J. Twin Higgs WIMP Dark Matter. Phys. Rev. D 2015, 92, 055034. [CrossRef]

152. Bai, Y.; Hill, R.J. Weakly Interacting Stable Pions. Phys. Rev. D 2010, 82, 111701. [CrossRef]

153. Bhattacharya, S.; Melić, B.; Wudka, J. Pionic Dark Matter. J. High Energy Phys. 2014, 02, 115. [CrossRef]

154. Hur, T.; Jung, D.W.; Ko, P.; Lee, J.Y. Electroweak symmetry breaking and cold dark matter from strongly interacting hidden sector. Phys. Lett. B 2011, 696, 262-265. [CrossRef]

155. Heikinheimo, M.; Racioppi, A.; Raidal, M.; Spethmann, C.; Tuominen, K. Physical Naturalness and Dynamical Breaking of Classical Scale Invariance. Mod. Phys. Lett. A 2014, 29, 1450077. [CrossRef]

156. Englert, C.; Jaeckel, J.; Khoze, V.V.; Spannowsky, M. Emergence of the Electroweak Scale through the Higgs Portal. J. High Energy Phys. 2013, 04, 060. [CrossRef]

157. Frigerio, M.; Pomarol, A.; Riva, F.; Urbano, A. Composite Scalar Dark Matter. J. High Energy Phys. 2012, 07, 015. [CrossRef]

158. Wess, J.; Zumino, B. Consequences of anomalous Ward identities. Phys. Lett. B 1971, 37, 95-97. [CrossRef]

159. Witten, E. Global Aspects of Current Algebra. Nucl. Phys. B 1983, 223, 422-432. [CrossRef]

160. Cai, H.; Cacciapaglia, G. Singlet dark matter in the SU(6)/SO(6) composite Higgs model. Phys. Rev. D 2021, 103, 055002. [CrossRef]

161. Alanne, T.; Gertov, H.; Sannino, F.; Tuominen, K. Elementary Goldstone Higgs boson and dark matter. Phys. Rev. D 2015, 91, 095021. [CrossRef]

162. Alanne, T.; Gertov, H.; Meroni, A.; Sannino, F. Vacuum alignment with and without elementary scalars. Phys. Rev. D 2016, 94 , 075015. [CrossRef]

163. Buckley, M.R.; Neil, E.T. Thermal dark matter from a confining sector. Phys. Rev. D 2013, 87, 043510. [CrossRef]

164. Alves, D.S.M.; Behbahani, S.R.; Schuster, P.; Wacker, J.G. Composite Inelastic Dark Matter. Phys. Lett. B 2010, 692, 323-326. [CrossRef]

165. Tucker-Smith, D.; Weiner, N. Inelastic dark matter. Phys. Rev. D 2001, 64, 043502. [CrossRef]

166. Tucker-Smith, D.; Weiner, N. The Status of inelastic dark matter. Phys. Rev. D 2005, 72, 063509. [CrossRef]

167. Chang, S.; Kribs, G.D.; Tucker-Smith, D.; Weiner, N. Inelastic Dark Matter in Light of DAMA/LIBRA. Phys. Rev. D 2009, 79, 043513. [CrossRef]

168. Kang, J.; Luty, M.A. Macroscopic Strings and 'Quirks' at Colliders. J. High Energy Phys. 2009, 11, 065. [CrossRef]

169. Peskin, M.E. The Alignment of the Vacuum in Theories of Technicolor. Nucl. Phys. B 1980, 175, 197-233. [CrossRef]

170. Harnik, R.; Kribs, G.D.; Martin, A. Quirks at the Tevatron and Beyond. Phys. Rev. D 2011, 84, 035029. [CrossRef]

171. Chivukula, R.S.; Walker, T.P. TECHNICOLOR COSMOLOGY. Nucl. Phys. B 1990, 329, 445-463. [CrossRef]

172. Gudnason, S.B.; Kouvaris, C.; Sannino, F. Dark Matter from new Technicolor Theories. Phys. Rev. D 2006, 74, 095008. [CrossRef]

173. Barr, S.M.; Chivukula, R.S.; Farhi, E. Electroweak Fermion Number Violation and the Production of Stable Particles in the Early Universe. Phys. Lett. B 1990, 241, 387-391. [CrossRef]

174. Kaplan, D.B. A Single explanation for both the baryon and dark matter densities. Phys. Rev. Lett. 1992, 68, 741-743. [CrossRef] [PubMed]

175. Faraggi, A.E.; Pospelov, M. Selfinteracting dark matter from the hidden heterotic string sector. Astropart. Phys. 2002, 16, 451-461. [CrossRef]

176. Lopez-Honorez, L.; Schwetz, T.; Zupan, J. Higgs portal, fermionic dark matter, and a Standard Model like Higgs at 125 GeV. Phys. Lett. B 2012, 716, 179-185. [CrossRef]

177. Djouadi, A.; Falkowski, A.; Mambrini, Y.; Quevillon, J. Direct Detection of Higgs-Portal Dark Matter at the LHC. Eur. Phys. J. C 2013, 73, 2455. [CrossRef]

178. Brooijmans, G.; Gripaios, B.; Moortgat, F.; Santiago, J.; Skands, P.; Albornoz Vásquez, D.; Allanach, B.C.; Alloul, A.; Arbey, A.; Azatov, A.; et al. Les Houches 2011: Physics at TeV Colliders New Physics Working Group Report. arXiv 2012, arXiv:1203.1488. 
179. Arcadi, G.; Covi, L. Minimal Decaying Dark Matter and the LHC. J. Cosmol. Astropart. Phys. 2013, 08, 005. [CrossRef]

180. Aaboud, M.; Aad, G.; Abbott, B.; Abdallah, J.; Abdinov, O.; Abeloos, B.; Aben, R.; AbouZeid, O.S.; Abraham, N.L.; Abramowicz, $\mathrm{H}$; ; et al. Search for heavy long-lived charged $R$-hadrons with the ATLAS detector in $3.2 \mathrm{fb}^{-1}$ of proton-proton collision data at $\sqrt{s}=13$ TeV. Phys. Lett. B 2016, 760, 647-665. [CrossRef]

181. Khachatryan, V.; Sirunyan, A.M.; Tumasyan, A.; Adam, W.; Asilar, E.; Bergauer, T.; Brandstetter, J.; Brondolin, E.; Dragicevic, M.; Erö, J.; et al. Search for long-lived charged particles in proton-proton collisions at $\sqrt{s}=13$ TeV. Phys. Rev. D 2016, 94,112004 . [CrossRef]

182. Molinaro, E.; Yaguna, C.E.; Zapata, O. FIMP realization of the scotogenic model. J. Cosmol. Astropart. Phys. 2014, $07,015$. [CrossRef]

183. Yaser Ayazi, S.; Firouzabadi, S.M.; Zakeri, S.P. Freeze-in production of Fermionic Dark Matter with Pseudo-scalar and Phenomenological Aspects. J. Phys. G 2016, 43, 095006. [CrossRef]

184. Hessler, A.G.; Ibarra, A.; Molinaro, E.; Vogl, S. Probing the scotogenic FIMP at the LHC. J. High Energy Phys. 2017, 01, 100. [CrossRef]

185. Ilnicka, A.; Robens, T.; Stefaniak, T. Constraining Extended Scalar Sectors at the LHC and beyond. Mod. Phys. Lett. A 2018, 33, 1830007. [CrossRef]

186. Aad, G.; Abbott, B.; Abdallah, J.; Abdinov, O.; Aben, R.; Abolins, M.; AbouZeid, O.S.; Abramowicz, H.; Abreu, H.; Abreu, R.; et al. Constraints on new phenomena via Higgs boson couplings and invisible decays with the ATLAS detector. J. High Energy Phys. 2015, 11, 206. [CrossRef]

187. Khachatryan, V.; Sirunyan, A.M.; Tumasyan, A.; Adam, W.; Asilar, E.; Bergauer, T.; Brandstetter, J.; Brondolin, E.; Dragicevic, M.; Erö, J.; et al. Searches for invisible decays of the Higgs boson in pp collisions at $\sqrt{s}=7,8$, and 13 TeV. J. High Energy Phys. 2017, 02, 135. [CrossRef]

188. Bernabei, R.; Belli, P.; Cappella, F.; Cerulli, R.; Dai, C.J.; d'Angelo, A.; He, H. L.;Incicchitti, A.; Kuang, H.H.; Ma, X.H.; et al. New results from DAMA/LIBRA. Eur. Phys. J. C 2010, 67, 39-49. [CrossRef]

189. Bernabei, R.; Belli, P.; Cappella, F.; Caracciolo, V.; Castellano, S.; Cerulli, R.; Dai, C.J.; d'Angelo, A.; d'Angelo, S.; Di Marco, A.; et al. Final model independent result of DAMA/LIBRA-phase1. Eur. Phys. J. C 2013, 73, 2648. [CrossRef]

190. Aalseth, C.E.; Barbeau, P.S.; Bowden, N.S.; Cabrera-Palmer, B.; Colaresi, J.; Collar, J. I.; Dazeley, S.; de Lurgio, P.; Fast, J.E.; Fields, N.; et al. Results from a Search for Light-Mass Dark Matter with a P-type Point Contact Germanium Detector. Phys. Rev. Lett. 2011, 106, 131301. [CrossRef]

191. Aalseth, C.E.; Barbeau, P.S.; Colaresi, J.; Collar, J.I.; Diaz Leon, J.; Fast, J.E.; Fields, N.; Hossbach, T.W.; Keillor, M.E.; Kephart, J.D.; et al. Search for an Annual Modulation in a P-type Point Contact Germanium Dark Matter Detector. Phys. Rev. Lett. 2011, 107, 141301. [CrossRef]

192. Aalseth, C.E.; Barbeau, P.S.; Colaresi, J.; Collar, J.I.; Leon, J.D.; Fast, J.E.; Fields, N.E.; Hossbach, T.W.; Knecht, A.; Kos, M.S.; et al. CoGeNT: A Search for Low-Mass Dark Matter using p-type Point Contact Germanium Detectors. Phys. Rev. D 2013, 88, 012002. [CrossRef]

193. Aalseth, C.E.; Barbeau, P.S.; Colaresi, J.; Collar, J.I.; Leon, J.D.; Fast, J.E.; Fields, N.E.; Hossbach, T.W.; Knecht, A.; Kos, M.S.; et al. Search for An Annual Modulation in Three Years of CoGeNT Dark Matter Detector Data. arXiv 2014, arXiv:1401.3295.

194. Aalseth, C.E.; Barbeau, P.S.; Colaresi, J.; Leon, J.D.; Fast, J.E.; Hossbach, T.W.; Knecht, A.; Kos, M.S.; Marino, M.G.; Miley, H.S.; et al. Maximum Likelihood Signal Extraction Method Applied to 3.4 years of CoGeNT Data. arXiv 2014, arXiv:1401.6234.

195. Agnese, R.; Ahmed, Z.; Anderson, A.J.; Arrenberg, S.; Balakishiyeva, D.; Thakur, R.B.; Bauer, D.A.; Billard, J.; Borgland, A.; Brandt, D.; et al. Silicon Detector Dark Matter Results from the Final Exposure of CDMS II. Phys. Rev. Lett. 2013, 111, 251301. [CrossRef]

196. Akerib, D.S.; Alsum, S.; Araújo, H.M.; Bai, X.; Bailey, A.J.; Balajthy, J.; Beltrame, P.; Bernard, E.P.; Bernstein, A.; Biesiadzinski, T.P.; et al. Results from a search for dark matter in the complete LUX exposure. Phys. Rev. Lett. 2017, 118, 021303. [CrossRef] [PubMed]

197. Aprile, E.; Aalbers, J.; Agostini, F.; Alfonsi, M.; Althueser, L.; Amaro, F.D.; Anthony, M.; Arneodo, F.; Baudis, L.; Bauermeister, B.; et al. Dark Matter Search Results from a One Ton-Year Exposure of XENON1T. Phys. Rev. Lett. 2018, 121, 111302. [CrossRef] [PubMed]

198. Ala-Mattinen, K.; Kainulainen, K. Precision calculations of dark matter relic abundance. J. Cosmol. Astropart. Phys. 2020, 09, 040. [CrossRef]

199. Arcadi, G.; Djouadi, A.; Kado, M. The Higgs-portal for dark matter: Effective field theories versus concrete realizations. Eur. Phys. J. C 2021, 81, 653. [CrossRef]

200. Alanne, T.; Benincasa, N.; Heikinheimo, M.; Kannike, K.; Keus, V.; Koivunen, N.; Tuominen, K. Pseudo-Goldstone dark matter: Gravitational waves and direct-detection blind spots. J. High Energy Phys. 2020, 10, 080. [CrossRef]

201. Alarcon, J.M.; Geng, L.S.; Martin Camalich, J.; Oller, J.A. The strangeness content of the nucleon from effective field theory and phenomenology. Phys. Lett. B 2014, 730, 342-346. [CrossRef]

202. Azevedo, D.; Duch, M.; Grzadkowski, B.; Huang, D.; Iglicki, M.; Santos, R. One-loop contribution to dark-matter-nucleon scattering in the pseudo-scalar dark matter model. J. High Energy Phys. 2019, 01, 138. [CrossRef]

203. Bulbul, E.; Markevitch, M.; Foster, A.; Smith, R.K.; Loewenstein, M.; Randall, S.W. Detection of An Unidentified Emission Line in the Stacked X-ray spectrum of Galaxy Clusters. Astrophys. J. 2014, 789, 13. [CrossRef]

204. Boyarsky, A.; Ruchayskiy, O.; Iakubovskyi, D.; Franse, J. Unidentified Line in X-Ray Spectra of the Andromeda Galaxy and Perseus Galaxy Cluster. Phys. Rev. Lett. 2014, 113, 251301. [CrossRef] [PubMed] 
205. Queiroz, F.S.; Sinha, K. The Poker Face of the Majoron Dark Matter Model: LUX to keV Line. Phys. Lett. B 2014, 735, 69-74. [CrossRef]

206. Baek, S.; Ko, P.; Park, W.I. The $3.5 \mathrm{keV}$ X-ray line signature from annihilating and decaying dark matter in Weinberg model. arXiv 2014, arXiv:1405.3730.

207. Farzan, Y.; Akbarieh, A.R. Decaying Vector Dark Matter as an Explanation for the $3.5 \mathrm{keV}$ Line from Galaxy Clusters. J. Cosmol. Astropart. Phys. 2014, 11, 015. [CrossRef]

208. Arcadi, G.; Covi, L.; Dradi, F. 3.55 keV line in Minimal Decaying Dark Matter scenarios. J. Cosmol. Astropart. Phys. 2015, $07,023$. [CrossRef]

209. Merle, A.; Schneider, A. Production of Sterile Neutrino Dark Matter and the $3.5 \mathrm{keV}$ line. Phys. Lett. B 2015, 749, $283-288$. [CrossRef]

210. Roland, S.B.; Shakya, B.; Wells, J.D. PeV neutrinos and a $3.5 \mathrm{keV}$ x-ray line from a PeV-scale supersymmetric neutrino sector. Phys. Rev. D 2015, 92, 095018. [CrossRef]

211. Kang, Z. Upgrading sterile neutrino dark matter to FImP using scale invariance. Eur. Phys. J. C 2015, 75, 471. [CrossRef]

212. Corbin, V.; Cornish, N.J. Detecting the cosmic gravitational wave background with the big bang observer. Class. Quant. Grav. 2006, 23, 2435-2446. [CrossRef]

213. Seto, N.; Kawamura, S.; Nakamura, T. Possibility of direct measurement of the acceleration of the universe using 0.1-Hz band laser interferometer gravitational wave antenna in space. Phys. Rev. Lett. 2001, 87, 221103. [CrossRef] 